\title{
How good are Portfolio Insurance Strategies?
}

\author{
Sven Balder $\ddagger$ \\ Antje Mahayni ${ }^{\ddagger \ddagger}$
}

This version: March 31, 2009

\begin{abstract}
Portfolio insurance strategies are designed to achieve a minimum level of wealth while at the same time participating in upward moving markets. The most prominent examples of dynamic versions are option based strategies with synthetic put and constant proportion portfolio insurance strategies. It is well known that, in a Black/Scholes type model setup, these strategies can be achieved as optimal solution by forcing an exogenously given guarantee into the expected utility maximization problem of an investor with CRRA utility function. The CPPI approach is attained by the introduction of a subsistence level, the OBPI approach stems from an additional constraint on the terminal portfolio value. We bring these results together in order to explain when and why OBPI strategies are better than CPPI strategies and vice versa. We determine the utility losses which are caused by introducing a terminal guarantee into the unconstrained maximization approach. In addition, we focus on utility losses which are due to market frictions such as discrete-time trading, transaction costs and borrowing constraints.
\end{abstract}

Keywords: CPPI, OBPI, portfolio insurance, borrowing constraints, trading restrictions, transaction costs, utility loss.

JEL: G11, G12

\footnotetext{
${ }^{\ddagger}$ Mercator School of Management, University of Duisburg-Essen, Lotharstr. 65, 47057 Duisburg, Germany. E-mail: sven.balder@uni-due.de

${ }^{\ddagger}$ Mercator School of Management, University of Duisburg-Essen, Lotharstr. 65, 47057 Duisburg, Germany. E-mail: antje.mahayni@uni-due.de
} 


\section{INTRODUCTION}

Portfolio strategies which are designed to limit downside risk and at the same time to profit from rising markets are summarized in the class of portfolio insurance strategies. Among others, Grossman and Villa (1989) and Basak (2002) define a portfolio insurance trading strategy as a strategy which guarantees a minimum level of wealth at a specified time horizon, but also participates in the potential gains of a reference portfolio. The most prominent examples of dynamic versions are the constant proportion portfolio insurance (CPPI) strategies and option-based portfolio insurance (OBPI) strategies with synthetic puts. ${ }^{1}$ The concept of (synthetic) option-based portfolio insurance is already introduced in Leland and Rubinstein (1976) and Brennan and Schwartz (1976). ${ }^{2}$ The constant proportion portfolio insurance (CPPI) is introduced in Black and Jones (1987). ${ }^{3}$

The popularity of portfolio insurance strategies can be explained by various reasons. On the side of institutional investors there are regulatory requirements including return guarantees as well as requisitions on the risk profile. For example, Ahn et al. (1999) consider the problem of an institution optimally managing the market risk of a given exposure by minimizing its Value-at-Risk using options. Amongst early papers on the optimality of portfolio insurance are also Leland (1980) and Benninga and Blume (1985). More recently, Doskeland and Nordahl (2008) justify the existence of guarantees from the point of an investor through behavioral models. In particular, they use cumulative prospect theory as an example. ${ }^{4}$

Unfortunately, the justification of guarantees is less clear assuming that the investor's preferences can be described using the von Neumann and Morgenstern (1944) framework of expected utility. Dating back to Merton (1971), it is well known that in a Black/Scholes model setup and a constant relative risk aversion (CRRA) utility function, the expected

\footnotetext{
${ }^{1}$ Principally, one can distinguish between two types of portfolio insurance strategies. A risky portfolio (or benchmark index) is combined either with a risk-free asset or with a financial derivative. In particular, the first class includes dynamic versions of OBPI, stop-loss strategies, buy and holds strategies and CPPI. The second class is mainly characterized by protective put strategies, either in a static or rolling sense. Notice that, with the exception of the buy and hold and the protective put, the above strategies are all dynamic in the sense that they afford portfolio adjustments during the investment horizon.

${ }^{2}$ For the evolution of portfolio insurance we refer to Leland and Rubinstein (1976)

${ }^{3}$ For the basic procedure of the CPPI see also Merton (1971).

${ }^{4}$ Basically, guarantees can be explained by a different treatment of gains and losses, i.e. losses are weighted more heavily than gains, cf. also Kahnemann and Tversky (1979) and Tversky and Kahnemann (1992).
} 
utility maximizing trading rule is a constant mix strategy, i.e. the fraction of asset exposure of the current wealth is to be kept constant over the time. In this case, an investment weight below one implies that assets are bought when the asset price decreases. This is in sharp contrast to portfolio insurance. In order to honor a terminal guarantee, the asset exposure is to be reduced if the price of the risky asset decreases. Technically, it is straightforward to achieve CPPI and OBPI strategies as the optimal solution of a modified utility maximization problem which is based on an exogenously given guarantee. CPPI strategies are optimal for an investor who derives utility from the difference between the terminal strategy value and a given subsistence level. In contrast, the OBPI is optimal for an CRRA investor if one exogenously adds the restriction that the terminal portfolio value is above the floor. ${ }^{5}$ These results are well known in the literature. Without postulating completeness, we refer to the works of Cox and Huang (1989), Brennan and Schwartz (1989), Grossman and Villa (1989), Black and Perold (1992), Grossman and Zhou (1993, 1996), Basak (1995), Cvitanic and Karatzas (1995, 1999), Browne (1999), Tepla (2000, 2001), Basak (2002) and El Karoui et al. (2005). Cox and Leland (2000) consider the inverse problem. They analyze if a specific dynamic strategy can be explained by solving the maximization problem of an expected utility maximizing investor, i.e. they analyze if a given investment strategy is consistent with expected utility maximization. In particular, they show that a strategy which implies a path-dependent payoff is not consistent with utility maximization in a Black/Scholes-type model.

Another strand of the literature analyzes robustness properties of stylized strategies. ${ }^{6}$ The properties of continuous-time CPPI strategies are studied extensively in the literature, cf. Bookstaber and Langsam (2000) or Black and Perold (1992). A comparison of OBPI and CPPI (in continuous time) is given in Bertrand and Prigent (2002a). Zagst and Kraus (2008) also compare OBPI and CPPI strategies. In particular, they derive parameter conditions implying second-and third-order stochastic dominance of the CPPI strategy. The literature also deals with the effects of jump processes, stochastic volatility models and extreme value approaches on the CPPI method, cf. Bertrand and Prigent (2002b), Bertrand and Prigent (2003). An analysis of gap risk, i.e. the risk that the guarantee is violated, is provided in Cont and Tankov (2007) and Balder et al. (2009). ${ }^{7}$

\footnotetext{
${ }^{5}$ In particular, the difference of OBPI and CPPI can be explained by the difference between postulating that the marginal utility jumps discontinuously to infinity or gradually, cf. for example Basak (2002).

${ }^{6} \mathrm{With}$ respect to the robustness of option hedges we refer the reader to Avellaneda et al. (1995), Lyons (1995), Bergman et al. (1996), El Karoui et al. (1998), Hobson (1998), Dudenhausen et al. (1998) and Mahayni (2003).

${ }^{7}$ Cont and Tankov (2007) introduce the gap risk by jump-diffusion models. Balder et al. (2009) introduce the gap by trading restrictions such that the analysis also captures the effects of transaction
} 
Finally, there is also a a wide strand of empirical papers which measure the performance of portfolio insurance strategies. For example, we refer to Cesari and Cremonini (2003) who give an extensive simulation comparison of popular dynamic strategies of asset allocation.

The following paper mitigates between expected utility maximization and the comparison of stylized strategies. We start with an exposition of the three optimization problems which imply constant mix, CPPI and OBPI strategies as optimal. Instead of giving a further justification for the existence of guarantees, we use the (well known) results of the optimization problems to explain the main differences between portfolio insurance mechanisms. Comparing the terminal payoffs shows that both portfolio insurance strategies, CPPI and OBPI, result in payoffs which consist of a fraction of the payoff of a constant mix strategy (which is optimal for the unconstrained CRRA investor) and an additional term due to the guarantee. The additional term provides an intuitive way to explain the main advantage of the OBPI approach as compared to the CPPI approach. Intuitively, it is clear that the fraction of wealth which is put into the optimal unconstrained stratgey is linked to the price of the guarantee, i.e. the fraction is less than one. In the case of the CPPI approach, the additional term is simply the guarantee itself, i.e. the payoff of an adequate number of zero bonds. In contrast, the additional term implied by the OBPI is a put option where the (synthetic) underlying is given by the fraction of constant mix strategy and the strike is equal to the guarantee. Obviously, the put is cheaper than the zero bonds. Therefore, an investor who follows the OBPI approach puts a larger fraction of his wealth into the unconstrained optimal portfolio than an investor who follows the CPPI approach. To asses the utility costs of forcing a guarantee into the unconstrained problem, i.e. the utility costs from having to use a suboptimal strategy, we compare the certainty equivalents of the different strategies and calculate the loss rates.

In addition, we explain one major drawback of the OBPI method which is due to the kink in the payoff-profile caused by the option component. The terminal value of the OBPI is equal to the guarantee if the put expires out of the money. In contrast to the CPPI method, this implies a positive point mass for the event that the terminal value is equal to the guarantee. The probability is given by the real world probability that the terminal asset prices is below the strike of the put. Intuitively, it is clear that this can cause a high exposure to gap risk, i.e. the risk that the guarantee is violated, if market

costs. In practice the gap risk was already observable during the 1987 crash. In addition, the crash is sometimes even explained or seen to be supported by the portfolio protection mechanisms. However, there are also contradicting opinions, cf. Leland (1988). The failure of the portfolio protection gave rise to a reduction in the application of portfolio insurance strategies. 
frictions are introduced. We illustrate this effect by taking trading restrictions and transaction costs into account. It turns out that the guarantee implied by the CPPI method is relatively robust. In contrast, the probability that the guarantee is not reached under the corresponding synthetic discrete-time OBPI strategy is rather high.

The outline of the paper is as follows. In Section 2, we review the well known optimization problems yielding constant mix, CPPI and OBPI strategies as optimal solutions. We compare the optimal strategies and resulting payoffs, and we discuss some advantages (disadvantages) of the different portfolio insurance methods. In Section 3, we consider the utility losses caused by the introduction of strictly positive terminal guarantees for a CRRA investor. In particular, we compare CPPI and OBPI strategies according to their implied loss rate. We consider the effects of market frictions in Section 4 where we focus on the loss rates which are implied by discrete-time trading and transaction costs. In addition, we compare the effects of these market frictions on the protection mechanisms of CPPI and OBPI. In Section 5, we address the topic of borrowing constrains and consider the capped version of CPPI strategies. Section 6 concludes the paper.

\section{Optimal Portfolio Selection with Finite Horizons}

All stochastic processes are defined on a stochastic basis $\left(\Omega, \mathcal{F},\left(\mathcal{F}_{t}\right)_{t \in\left[0, T^{*}\right]}, P\right)$ which satisfies the usual hypotheses. We consider two assets. The riskless bond $B$ grows at a constant interest rate $r$, i.e. $d B_{t}=B_{t} r d t$ where $B_{0}=b$. The evolution of the risky asset $S$, a stock or benchmark index, is given by a geometric Brownian motion

$$
d S_{t}=S_{t}\left(\mu d t+\sigma d W_{t}\right), \quad S_{0}=s
$$

where $W=\left(W_{t}\right)_{0 \leq t \leq T}$ denotes a standard Brownian motion with respect to the real world measure $P$. $\mu$ and $\sigma$ are constants and we assume that $\mu>r \geq 0$ and $\sigma>0$.

A continuous-time investment strategy or saving plan for the interval $[0, T]$ can be represented by a predictable process $\left(\pi_{t}\right)_{0 \leq t \leq T} . \pi_{t}$ denotes the proportion of the portfolio value at time $t$ which is invested in the risky asset $S$. In the following, we also refer to $\pi_{t}$ as the portfolio weight at time $t$. W.l.o.g., we consider strategies which are self-financing, i.e. money is neither injected nor withdrawn during the investment horizon $[0, T]$. Thus, the fraction of wealth which is invested at time $t$ in the riskless bond $B$ is given by $1-\pi_{t}$.

Let $V=\left(V_{t}\right)_{0 \leq t \leq T}$ denote the portfolio value process associated with the strategy $\pi$, then the dynamics of $V$ are given by

$$
d V_{t}(\pi)=V_{t}\left(\pi_{t} \frac{d S_{t}}{S_{t}}+\left(1-\pi_{t}\right) \frac{d B_{t}}{B_{t}}\right) \text {, where } V_{0}=x .
$$


For the above model assumptions, it follows

$$
d V_{t}(\pi)=V_{t}\left[\left(\pi_{t}(\mu-r)+r\right) d t+\pi_{t} \sigma d W_{t}\right] \text {, where } V_{0}=x .
$$

Alternatively, the strategies can be represented by the number of shares. Let $\rho_{t}=$ $\left(\rho_{t, S}, \rho_{t, B}\right)_{0 \leq t \leq T}$ where $\rho_{t, S}$ denotes the number of risky assets and $\rho_{t, B}$ the number of zero bonds with maturity $T$ which are held at time $t$. In particular, we have

$$
\rho_{t, S}=\frac{\pi_{t} V_{t}}{S_{t}} \text { and } \rho_{t, B}=\frac{\left(1-\pi_{t}\right) V_{t}}{B_{t}}
$$

where $B_{t}$ denotes the $t$-price of the zero bond maturing at $T$. Traditionally, a strategy specification via the portfolio weights is used in the context of portfolio optimization while the convention of stating the number of shares is normally preferred in the context of hedging.

In the case of a finite investment horizon $T$ and no intermediate consumption possibilities, the relevant optimization problem is given by

$$
\sup _{\pi \in \Pi} \mathbb{E}_{P}\left[u\left(V_{T}(\pi)\right)\right] \text { subject to Equation (3), }
$$

where $\Pi$ denotes the set of all self-financing trading strategies. The utility function $u$ $\left(u \in C^{2}\right)$ is assumed to be strictly increaing and concave, i.e. $u^{\prime}>0$ and $u^{\prime \prime}<0$. In the following, we recall the well known optimization problems which justify three basic strategy classes: constant mix (CM) strategies, constant proportion portfolio insurance strategies (CPPI) and option based strategies (OBPI). In contrast to a CM strategy which is exclusively specified by a constant portfolio weight $m$, i.e. $\pi_{t}^{C M}=m$, portfolio insurance strategies incorporate a guarantee component which, in the simplest case, is given by an amount $G_{T}$ which is to be honored at the end of the investment horizon $T$. While a CPPI strategy is value based in the sense that the portfolio weights are exclusively specified by the current portfolio value (and the present value of the guarantee), the (dynamic) OBPI approach is payoff and model dependent. Here, the investment decisions are, in a complete model, given in terms of the delta hedge of an option payoff. Formally, the three strategy classes are:

$$
\begin{aligned}
\Pi^{C M}= & \left\{\pi \in \Pi \mid \pi_{t}=m, m \geq 0\right\} \\
\Pi^{C P P I_{G}}= & \left\{\pi \in \Pi \mid \pi_{t}=m \frac{V_{t}-e^{-r(T-t)} G_{T}}{V_{t}}, m \geq 0\right\} \\
\Pi^{O B P I_{G}}= & \left\{\pi \in \Pi \mid \pi_{t}=\frac{\Delta_{t} S_{t}}{V_{t}}, \Delta_{t}=\frac{\partial}{\partial S_{t}} \mathbb{E}_{P^{*}}\left[e^{-r(T-t)}\left(h\left(S_{T}\right)-G_{T}\right)^{+} \mid \mathcal{F}_{t}\right],\right. \\
& \left.h \in C^{2},\left(\frac{\partial P^{*}}{\partial P}\right)_{T}=e^{-\frac{1}{2}\left(\frac{\mu-r}{\sigma}\right)^{2} T-\frac{\mu-r}{\sigma} W_{T}}\right\} .
\end{aligned}
$$


Optimization problems

\begin{tabular}{|c|c|c||c|}
\hline problem & utility function $(\gamma>0, \gamma \neq 1)$ & additional constraint & optimal strategy \\
\hline$(\mathrm{A})$ & $u_{A}\left(V_{T}\right)=\frac{V_{T}^{1-\gamma}}{1-\gamma}$ & none & CM \\
$(\mathrm{B})$ & $u_{B}\left(V_{T}\right)=\frac{\left(V_{T}-G_{T}\right)^{1-\gamma}}{1-\gamma}$ & none & CPPI \\
$(\mathrm{C})$ & $u_{A}\left(V_{T}\right)=\frac{V_{T}^{1-\gamma}}{1-\gamma}$ & $V_{T} \geq G_{T}$ & OBPI \\
\hline
\end{tabular}

TABLE 1. Benchmark optimization problems.

Notice that for $G_{T}=0$, we have $\Pi^{C M}=\Pi^{C P P I_{G}}$. However, we refer to CPPI and OBPI versions where the guarantee is not a strategy parameter but $G_{T}>0$ is exogenously given. In particular, we assume that $G_{T}<V_{0} e^{r T}$.

Table 1 summarizes the optimization problems which are suited to justify the three strategy classes. Problem (A) is the classic Merton problem, cf. Merton (1971). Problem (B) introduces a subsistence level $G_{T}$ such that $u_{B}$ belongs to the class of HARA utility functions. Problem (C) consists of the CRRA utility function $u_{A}$ which is also used in problem (A) but poses an additional constraint on the terminal value of the strategy, i.e. the constraint that the terminal strategy value must be above or equal to the terminal guarantee $G_{T}$. The solutions of the optimization problems and their corresponding proofs are well known in the literature such that we omit some technical parts of the proofs and refer to the literature given in the introduction.

Intuitively, it is clear that the solutions of problems (B) and (C) are modifications of the classic Merton problem where a guarantee is exogenously forced into the optimization problem, respectively the solution. Basically, the subsistence level in (B) results in the optimization problem of $(\mathrm{A})$ if the value process is reduced by the present value of the terminal guarantee, i.e. the optimization problem (A) is given in terms of the cushion process. Technically, the solution of problem (C) is more involved. However, the solution of $(\mathrm{C})$ is intuitive in the sense that the constraint on the terminal value features an European option on the optimal payoff of $(\mathrm{A})$ where the initial investment must take into account the price of the option.

2.1. Problem (A). The basic observation which simplifies the optimization problem (A) to a large extend is the independence of the optimal portfolio weight of the investment horizon $T$ and the current asset price $S_{0}$. This implies that the solution of problem (A) can be obtained by restricting the strategy set to constant mix (CM) strategies such that 
it is enough to consider the maximization problem

$$
\sup _{\pi \in \Pi^{C M}} \mathbb{E}_{P}\left[u_{A}\left(V_{T}(\pi)\right)\right] \text { subject to Equation (3). }
$$

Notice that for $\pi \in \Pi^{C M}$, Equation (3) simplifies to

$$
\begin{aligned}
d V_{t}^{C M} & =V_{t}^{C M}\left[(r+m(\mu-r)) d t+m \sigma d W_{t}\right] \\
\text { i.e. } \quad V_{T}^{C M} & =V_{0}^{C M} e^{\left(r+m(\mu-r)-\frac{1}{2} m^{2} \sigma^{2}\right) T+m \sigma W_{T}} .
\end{aligned}
$$

Inserting $\sigma W_{T}=\ln \frac{S_{T}}{S_{0}}-\left(\mu-\frac{1}{2} \sigma^{2}\right) T$ gives $^{8}$

$$
\begin{aligned}
V_{T}^{\mathrm{CM}} & =V_{0}^{C M} e^{\left(m(\mu-r)+r-\frac{1}{2} m^{2} \sigma^{2}\right) T-m\left(\mu-\frac{1}{2} \sigma^{2}\right) T}\left(\frac{S_{T}}{S_{0}}\right)^{m} \\
& =\phi\left(V_{0}^{C M}, m\right) S_{T}^{m} \\
\text { where } \phi(x, y) & :=x\left(\frac{1}{S_{0}}\right)^{y} e^{(1-y)\left(r+\frac{1}{2} y \sigma^{2}\right) T} .
\end{aligned}
$$

The expected utility is equal to

$$
\begin{aligned}
\mathbb{E}_{P}\left[u_{A}\left(V_{T}^{\mathrm{CM}}\right)\right] & =\frac{\phi\left(V_{0}^{C M}, m\right)^{1-\gamma}}{1-\gamma} \mathbb{E}\left[S_{T}^{(1-\gamma) m}\right] \\
& =\frac{\left(V_{0}^{C M}\right)^{1-\gamma}}{1-\gamma} e^{(1-\gamma)\left(r+m(\mu-r)-\frac{1}{2} \gamma m^{2} \sigma^{2}\right) T}
\end{aligned}
$$

Finally, it is straightforward to show that

$$
\operatorname{argmax}_{m} \mathbb{E}_{P}\left[u_{A}\left(V_{T}^{\mathrm{CM}}\right)\right]=\frac{\mu-r}{\gamma \sigma^{2}}=: m^{*}
$$

2.2. Problem (B). Consider now the (modified) portfolio planning problem of an investor who derives utility from the difference between the portfolio value and a given subsistence level. Let $C=\left(C_{t}\right)_{0 \leq t \leq T}$ denote the cushion process where $C_{t}:=V_{t}-e^{-r T} G_{T}$. If a constant proportion $m$ of the cushion is invested in the risky asset, one obtains analogously to the Equations (9) and (11)

$$
\begin{aligned}
d C_{t} & =C_{t}\left[(r+m(\mu-r)) d t+m \sigma d W_{t}\right] \\
\text { and } C_{T} & =\phi\left(C_{0}, m\right) S_{T}^{m}
\end{aligned}
$$

as well as

$$
\operatorname{argmax}_{m} \mathbb{E}_{P}\left[u_{A}\left(C_{T}\right)\right]=\operatorname{argmax}_{m} \mathbb{E}_{P}\left[u_{A}\left(\phi\left(C_{0}, m\right) S_{T}^{m}\right)\right]=\frac{\mu-r}{\gamma \sigma^{2}}=m^{*} .
$$

\footnotetext{
${ }^{8}$ Notice that, as a function of the terminal asset price $S_{T}$, the payoff $V_{T}^{C M}$ is concave for $m<1$, linear for $m=1$ and convex for $m>1$.
} 
Notice that $E_{P}\left[u_{A}\left(C_{T}\right)\right]=E_{P}\left[u_{B}\left(V_{T}\right)\right]$. Together with the result that the optimal payoff does not depend on the asset price path implies that the optimal investment proportion $\pi_{t, B}^{*}$ of problem (B) is ${ }^{9}$

$$
\pi_{t, B}^{*}=\frac{m^{*} C_{t}}{V_{t}}=\frac{m^{*}\left(V_{t}-e^{-r(T-t)} G_{T}\right)}{V_{t}} .
$$

Obviously, problem (A) and (B) coincide in the case that $G_{T}=0$. However, $G_{T}>0$ implies a reduction in the investment proportion, i.e. ${ }^{10}$

$$
\pi_{t, B}^{*} \leq m^{*}=\pi_{t, A}^{*}
$$

Thus, $\pi_{t, B}^{*}$ is suboptimal with respect to the problem setup (A). In particular, the reduction in the expected utility can be interpreted as the utility loss arising from the guarantee component $G_{T}>0$.

2.3. Problem (C). The additional constraint $V_{T} \geq G_{T}$ together with the path-independency of the optimal solution implies that the optimal payoff $V_{T, C}^{*}$ can be represented as follows

$$
V_{T, C}^{*}=\max \left\{h\left(S_{T}\right), G_{T}\right\}=h\left(S_{T}\right)+\left[G_{T}-h\left(S_{T}\right)\right]^{+}=G_{T}+\left[h\left(S_{T}\right)-G_{T}\right]^{+} .
$$

Thus, the terminal value of the strategy can be interpreted in terms of an option on the payoff $h$ such that the optimal strategy $\pi^{*}$ is given in terms of the delta hedge, i.e. $\pi^{*} \in \Pi^{O B P I}$. In the case that $G_{T}=0$, the optimization problem reduces to the classic Merton case, i.e. for $G_{T}=0$ it holds $h\left(S_{T} ; G_{T}=0\right)=\phi\left(V_{0}, m^{*}\right) S_{T}^{m^{*}}$. For $G_{T}>0$ (and $T>0$ ), the optimal solution affords a reduction of the initial investment from $V_{0}$ to $\tilde{V}_{0}\left(\tilde{V}_{0}<V_{0}\right)$ such that $h\left(S_{T}\right)=\phi\left(\tilde{V}_{0}, m^{*}\right) S_{T}^{m^{*}}$. The remaining money $V_{0}-\tilde{V}_{0}$ is then used to buy the put with payoff $\left[G_{T}-h\left(S_{T}\right)\right]^{+}$. A proof for the optimality of the payoff $V_{T, C}^{*}=G_{T}+\left[\phi\left(\tilde{V}_{0}, m^{*}\right) S_{T}^{m^{*}}-G_{T}\right]^{+}$is given in El Karoui et al. (2005). The concavity of $u_{A}(x)=\frac{x^{1-\gamma}}{1-\gamma}$ implies that for any payoff $\hat{V}_{T}$ with $\hat{V}_{T} \geq G_{T}$

$$
\begin{aligned}
& u_{A}\left(\hat{V}_{T}\right)-u_{A}\left(V_{T, C}^{*}\right) \leq u_{A}^{\prime}\left(V_{T, C}^{*}\right)\left(\hat{V}_{T}-V_{T, C}^{*}\right) \\
& =\left\{\begin{array}{ll}
\phi\left(\tilde{V}_{0}, m^{*}\right)^{-\gamma} S_{T}^{-\gamma m^{*}}\left(\hat{V}_{T}-V_{T, C}^{*}\right) & \text { for } S_{T}^{m^{*}}>\frac{G_{T}}{\phi\left(\tilde{V}_{0}, m^{*}\right)} \\
G_{T}^{-\gamma}\left(\hat{V}_{T}-G_{T}\right) & \text { for } S_{T}^{m^{*}} \leq \frac{G_{T}}{\phi\left(\tilde{V}_{0}, m^{*}\right)}
\end{array},\right.
\end{aligned}
$$

i.e.

$u_{A}\left(\hat{V}_{T}\right)-u_{A}\left(V_{T, C}^{*}\right) \leq \phi\left(\tilde{V}_{0}, m^{*}\right)^{-\gamma} S_{T}^{-\gamma m^{*}}\left(\hat{V}_{T}-V_{T, C}^{*}\right)-\left(\phi\left(\tilde{V}_{0}, m^{*}\right)^{-\gamma} S_{T}^{-\gamma m^{*}}-G_{T}^{-\gamma}\right)^{+}\left(\hat{V}_{T}-G_{T}\right)$

\footnotetext{
${ }^{9}$ For the path independence cf. for example Cox and Leland (2000).

${ }^{10}$ The cushion dynamics given by Equation (15) immediately implies $V_{t}=e^{-r(T-t)} G_{T}+\phi\left(C_{0}, m ; t\right) S_{t}^{m}$, i.e. $V_{t} \geq e^{-r(T-t) G_{T}}$.
} 
Consider the first term on the right hand side of the above inequality. Adding and subtracting the optimal unconstrained solution $V_{T, A}^{*}$ and taking expectations gives ${ }^{11}$

$$
\phi\left(\tilde{V}_{0}, m^{*}\right)^{-\gamma}\left(\mathbb{E}\left[S_{T}^{-\gamma m^{*}}\left(\hat{V}_{T}-V_{T, A}^{*}\right]+\mathbb{E}\left[S_{T}^{-\gamma m^{*}}\left(V_{T, A}^{*}-V_{T, C}^{*}\right)\right]\right)=0 .\right.
$$

Together with $\hat{V}_{T} \geq G_{T}$ a.s. it follows $\mathbb{E}\left[u_{A}\left(\hat{V}_{T}\right)-u_{A}\left(V_{T, C}^{*}\right)\right] \leq 0$ such that $V_{T, C}^{*}$ is indeed the optimal solution w.r.t. problem $(\mathrm{C})$.

The payoff $V_{T, C}^{*}=G_{T}+\left[\phi\left(\tilde{V}_{0}, m^{*}\right) S_{T}^{m^{*}}-G_{T}\right]^{+}$can be replicated by a self-financing strategy where the initial investment can be represented by the expected discounted payoff under the uniquely defined equivalent martingale measure $P^{*}$ defined in Equation (8), i.e.

$$
\begin{aligned}
V_{0} & =e^{-r T} \mathbb{E}_{P^{*}}\left[G_{T}+\left(\phi\left(\tilde{V}_{0}, m^{*}\right) S_{T}^{m^{*}}-G_{T}\right)^{+}\right] \\
& =e^{-r T} G_{T}+\phi\left(\tilde{V}_{0}, m^{*}\right) \mathbb{E}_{P^{*}}\left[e^{-r T}\left(S_{T}^{m^{*}}-\frac{G_{T}}{\phi\left(\tilde{V}_{0}, m^{*}\right)}\right)^{+}\right] .
\end{aligned}
$$

$\tilde{V}_{0}$ has to be determined such that the initial cushion $C_{0}=V_{0}-e^{-r T} G_{T}$ exactly finances $\phi\left(\tilde{V}_{0}, m^{*}\right)$ power call options with power $p=m^{*}$ and strike $K=\frac{G_{T}}{\phi\left(\tilde{V}_{0}, m^{*}\right)}$, i.e.

$$
V_{0}-e^{-r T} G_{T}=\phi\left(\tilde{V}_{0}, m^{*}\right) P O\left(0, S_{0} ; m^{*}, \frac{G}{\phi\left(\tilde{V}_{0}, m^{*}\right)}\right)
$$

where $P O\left(t, S_{t} ; p, K\right)$ denotes the $t$-price a power call with power $p$, strike $K$ and maturity T, i.e. ${ }^{12}$

$$
\begin{gathered}
P O\left(t, S_{t} ; p, K\right):=e^{-r(T-t)} \mathbb{E}_{P^{*}}\left[\left(S_{T}^{p}-K\right)^{+} \mid \mathcal{F}_{t}\right] \\
=e^{-r(T-t)}\left[\left(\frac{S_{t}}{e^{-r(T-t)}}\right)^{p} e^{-\frac{1}{2} p(1-p) \sigma^{2}(T-t)} \mathcal{N}\left(h_{1}\left(t, \frac{S_{t}}{e^{-r(T-t)} \sqrt[p]{K}}\right)-(1-p) \sigma \sqrt{T-t}\right)\right. \\
\left.-K \mathcal{N}\left(h_{2}\left(t, \frac{S_{t}}{e^{-r(T-t)} \sqrt[p]{K}}\right)\right)\right]
\end{gathered}
$$

${ }^{11}$ Consider the portfolio $V_{T}(\varepsilon)=\varepsilon V_{T, A}^{*}+(1-\varepsilon) \tilde{V}_{T}$. The first order condition of optimization problem (A) implies

$$
\left.\frac{\partial \mathbb{E}\left[u\left(V_{T}(\varepsilon)\right)\right]}{\partial \varepsilon}\right|_{\varepsilon=1}=\left.\mathbb{E}\left[u^{\prime}\left(V_{T}(\varepsilon)\right)\left(V_{T, A}^{*}-\tilde{V}_{T}\right)\right]\right|_{\varepsilon=1}=\mathbb{E}\left[\phi\left(V_{0}, m^{*}\right)^{-\gamma} S_{T}^{-\gamma m^{*}}\left(V_{T, A}^{*}-\tilde{V}_{T}\right)\right]=0 .
$$

${ }^{12}$ The pricing formula is well known in the literature, cf. Zhang (1998), p. 597, Equation (30.3). The proof is easily done by using a change of measure, cf. for example Esser (2003) or Mahayni and Schlögl (2008). 
$\mathcal{N}$ denotes the one-dimensional standard normal distribution function. The functions $h_{1}$ and $h$, are given by

$$
h_{1}(t, z)=\frac{\ln z+\frac{1}{2} \sigma^{2}(T-t)}{\sigma \sqrt{T-t}} ; \quad h_{2}(t, z)=h_{1}(t, z)-\sigma \sqrt{T-t} .
$$

Recall that $\tilde{V}_{0} \leq V_{0}$. A lower bound on $\tilde{V}_{0}$ follows with

$$
\begin{gathered}
{\left[S_{T}^{m^{*}}-\frac{G_{T}}{\phi\left(\tilde{V}_{0}, m^{*}\right)}\right]^{+} \leq S_{T}^{m^{*}}} \\
\Rightarrow \phi\left(\tilde{V}_{0}, m^{*}\right) \operatorname{PO}\left(0, S_{0} ; m^{*}, \frac{G}{\phi\left(\tilde{V}_{0}, m^{*}\right)}\right) \leq \phi\left(\tilde{V}_{0}, m^{*}\right) \mathbb{E}_{P^{*}}\left[e^{-r T} S_{T}^{m^{*}}\right]=\tilde{V}_{0}
\end{gathered}
$$

and Equation (22). This implies

$$
V_{0}-e^{-r T} G_{T} \leq \tilde{V}_{0} \leq V_{0} .
$$

Consider now the optimal portfolio weight $\pi_{t, C}^{*}$ of the dynamic strategy, i.e.

$$
\begin{aligned}
\pi_{t, C}^{*} & =\frac{\phi\left(\tilde{V}_{0}, m^{*}\right) \Delta^{P O}\left(t, S_{t} ; m^{*}, \frac{G_{T}}{\phi\left(\tilde{V}_{0}, m^{*}\right)}\right) S_{t}}{V_{t}} \\
& =\frac{\phi\left(\tilde{V}_{0}, m^{*}\right) \Delta^{P O}\left(t, S_{t} ; m^{*}, \frac{G_{T}}{\phi\left(\tilde{V}_{0}, m^{*}\right)}\right) S_{t}}{e^{-r(T-t)} G_{T}+\phi\left(\tilde{V}_{0}, m^{*}\right) P O\left(t, S_{t} ; m^{*}, \frac{G}{\phi\left(\tilde{V}_{0}, m^{*}\right)}\right)}
\end{aligned}
$$

where $\Delta_{t}^{P O}:=\frac{\partial P O}{\partial S_{t}}\left(t, S_{t} ; p, K\right)$. Differentiating (23) immediately gives

$$
\begin{aligned}
\Delta^{P O}\left(t, S_{t} ; p, K\right)= & p\left(\frac{S_{t}}{e^{-r(T-t)}}\right)^{p-1} \\
& e^{-\frac{1}{2} p(1-p) \sigma^{2}(T-t)} \mathcal{N}\left(h_{1}\left(t, \frac{S_{t}}{e^{-r(T-t)} \sqrt[p]{K}}\right)-(1-p) \sigma \sqrt{T-t}\right) \cdot
\end{aligned}
$$

Finally, we compare the optimal investment proportion implied by problem $(\mathrm{C})$ with the one of the unconstrained solution given by $m^{*}$. Notice that

$$
\left[S_{T}^{m^{*}}-\frac{G_{T}}{\phi\left(\tilde{V}_{0}, m^{*}\right)}\right]^{+} \geq S_{T}^{m^{*}}-\frac{G_{T}}{\phi\left(\tilde{V}_{0}, m^{*}\right)}
$$

implies that the denominator of the right hand side of Equation (26) is larger than

$$
\phi\left(\tilde{V}_{0}, m^{*}\right) \mathbb{E}_{P^{*}}\left[e^{-r(T-t)} S_{T}^{m^{*}}\right]=\phi\left(\tilde{V}_{0}, m^{*}\right) S_{t}^{m^{*}} e^{\left(m^{*}-1\right)\left(r+\frac{1}{2} m^{*} \sigma^{2}\right)(T-t)}
$$

such that

$$
\pi_{t, C}^{*} \leq \Delta^{P O}\left(t, S_{t} ; m^{*}, \frac{G_{T}}{\phi\left(\tilde{V}_{0}, m^{*}\right)}\right) S_{t}^{\left(1-m^{*}\right)} e^{\left(1-m^{*}\right)\left(r+\frac{1}{2} m^{*} \sigma^{2}\right)(T-t)} .
$$

In addition, Equation (27) immediately gives

$$
\Delta^{P O}\left(t, S_{t} ; m^{*}, \frac{G_{T}}{\phi\left(\tilde{V}_{0}, m^{*}\right)}\right) \leq m^{*}\left(\frac{S_{t}}{e^{-r(T-t)}}\right)^{m^{*}-1} e^{-\frac{1}{2} m^{*}\left(1-m^{*}\right) \sigma^{2}(T-t)}
$$


Basic parameter constellation

\begin{tabular}{|l|l|l|}
\hline model paramter & strategy parameter & terminal guarantee \\
\hline$S_{0}=1$ & $V_{0}=1$ & $G_{T}=1$ \\
$\sigma=0.15$ & $T=10$ & \\
$r=0.03$ & $\gamma=1.2$ & \\
$\mu=0.085$ & $m=m^{*}=2.037$ & \\
\hline
\end{tabular}

TABLE 2. Basic parameter constellation.

\section{Optimal payoffs}

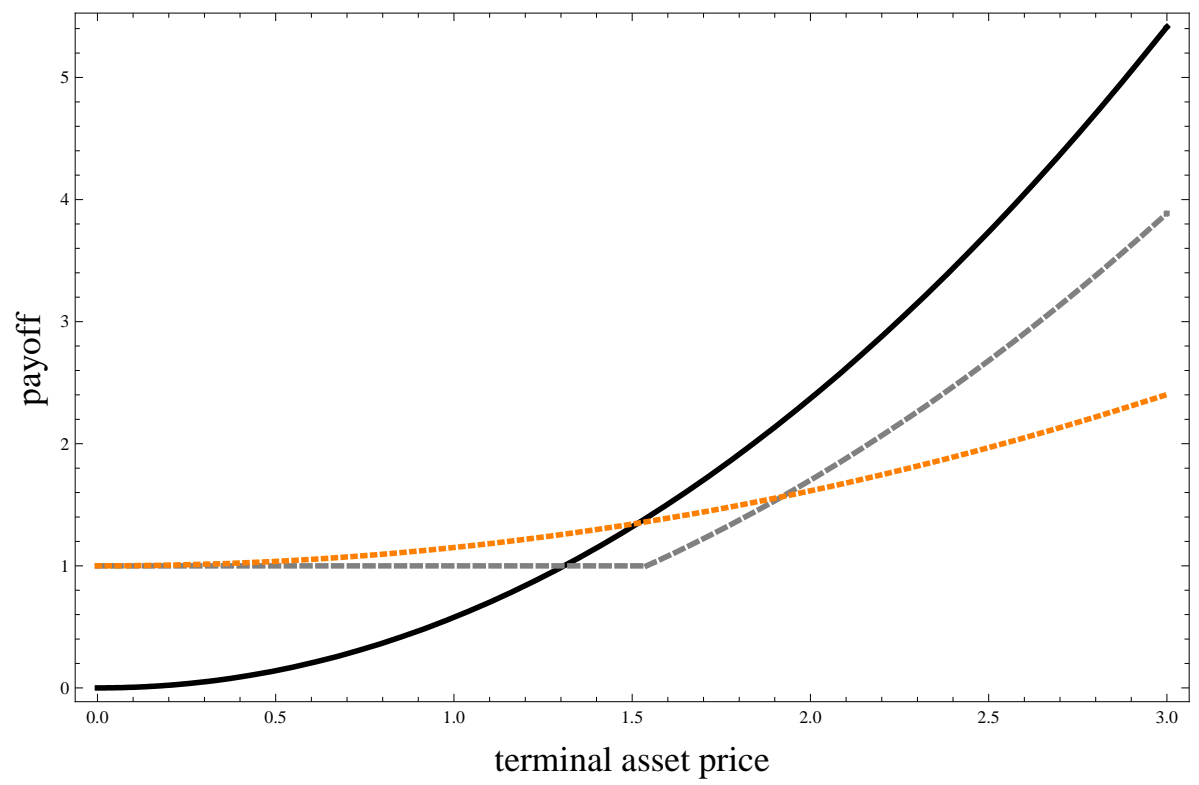

Figure 1. Optimal payoffs $V_{T, A}^{*}$ (solid line), $V_{T, B}^{*}$ (dotted line) and $V_{T, C}^{*}$ (dashed line) where the parameters are given as in Table 2.

Together, we have

$$
\pi_{t, C}^{*} \leq m^{*}
$$

Analogously to the problem (B), the terminal constraint in problem (C) also gives rise to a reduction of the optimal unconstrained portfolio weight $m^{*}$.

2.4. Comparison of optimal solutions. Recall that the optimal terminal payoffs $V_{T}^{*}$ do not depend on the asset price path such that they can be exclusively specified in the terminal asset price $S_{T} \cdot{ }^{13}$ The optimal payoffs for the optimization problems (A), (B)

\footnotetext{
${ }^{13}$ In particular, the payoffs are concave for $m<1$, linear for $m=1$ and convex for $m>1$.
} 
and $(\mathrm{C})$ are summarized as follows

$$
\begin{aligned}
V_{T, A}^{*} & =\phi\left(V_{0}, m^{*}\right) S_{T}^{m^{*}} \\
V_{T, B}^{*} & =G_{T}+\phi\left(V_{0}-e^{-r T} G_{T}, m^{*}\right) S_{T}^{m^{*}}=G_{T}+\frac{V_{0}-e^{-r T} G_{T}}{V_{0}} V_{T, A}^{*} \\
\text { and } V_{T, C}^{*} & =G_{T}+\left[\phi\left(\tilde{V}_{0}, m^{*}\right) S_{T}^{m^{*}}-G_{T}\right]^{+} \\
& =\phi\left(\tilde{V}_{0}, m^{*}\right) S_{T}^{m^{*}}+\left[G_{T}-\phi\left(\tilde{V}_{0}, m^{*}\right) S_{T}^{m^{*}}\right]^{+} \\
& =\frac{\tilde{V}_{0}}{V_{0}} V_{T, A}^{*}+\left[G_{T}-\frac{\tilde{V}_{0}}{V_{0}} V_{T, A}^{*}\right]^{+} .
\end{aligned}
$$

The payoff $V_{T, A}^{*}$ corresponds to $\phi\left(V_{0}, m^{*}\right)$ power claims with power $m^{*}$ where the number $\phi\left(V_{0}, m^{*}\right)$ depends on the initial investment and the optimal investment proportion $m^{*}$. The optimization problem (B) introduces a subsistence level which implies that the number of power claims with power $m^{*}$ must be reduced to afford the risk-free investment which is necessary to honor the guarantee. In consequence the portfolio weight is lower than in the case of problem (A), cf. Inequality (19). The link between the solutions of (A) and (B) is even more explicit if one considers the number of shares in the asset which are held. Notice that the cushion dynamics, cf. Equation (15) implies that $\frac{C_{t}^{C P P I}}{C_{0}^{C P P I}}=\frac{V_{t}^{C M}}{V_{0}}$ if the multiplier $m$ of the CPPI is equal to the portfolio weight of the CM strategy. In particular, this implies that the value of the cushion is proportional to the value of the CM strategy, i.e. $C_{t}^{C P P I}=\frac{C_{0}}{V_{0}} V_{t}^{C M}$. This is also true for the number of assets $\rho_{t, S}$, i.e.

$$
\begin{aligned}
\rho_{t, S}^{C P P I} & =\frac{C_{0}}{V_{0}} \rho_{t, S}^{C M} \\
\rho_{t, B}^{C P P I} & =G_{T}+\frac{C_{0}}{V_{0}} \rho_{t, B}^{C M}
\end{aligned}
$$

where $\rho_{t, B}$ denotes the number of zero bonds with maturity $T$. In particular, it holds that

$$
V_{t}^{C P P I}=e^{-r(T-t)} G_{T}+\frac{C_{0}}{V_{0}} V_{t}^{C M} .
$$

The CPPI strategy can thus be interpreted as a buy and hold strategy of a constant mix strategy with an additional investment into $G_{T}$ zero bonds, cf. also Equation (31). A similar reasoning applies to the solution of $(\mathrm{C})$ which can be interpreted as a buy and hold strategy of a constant mix strategy with an additional investment into a put with strike $G_{T}$, cf. Equation (33). Obviously, the put is worth less than $G_{T}$ zero bonds such that one can buy and hold more CM strategies in the case of the option based approach, i.e. $\frac{\tilde{V}_{0}}{V_{0}} \geq \frac{C_{0}}{V_{0}}$. Intuitively, it is thus clear that the OBPI approach gives a better result than the CPPI approach with respect to a utility function which favors the CM strategy with portfolio weight $m^{*}$. 
In general, a modification of the payoff $V_{T, A}^{*}$ which honors the guarantee $G_{T}$ and with $t_{0}$-price equal to $V_{0}$ can be represented by

$$
G_{T}+\alpha\left[V_{T, A}^{*}-\beta \frac{G_{T}}{\alpha}\right]^{+} \text {subject to } \mathbb{E}_{P^{*}}\left[e^{-r T}\left(G_{T}+\alpha\left[V_{T, A}^{*}-\beta \frac{G_{T}}{\alpha}\right]^{+}\right)\right]=V_{0}
$$

The CPPI approach corresponds to $\beta=0$ and gives a smooth payoff-profile. $\beta=1$ results in the OBPI approach with a kinked payoff-profile. As a consequence of the guarantee $G_{T}$, both payoffs $V_{T, B}^{*}$ and $V_{T, C}^{*}$ are higher (lower) than $V_{T, A}^{*}$ for low (high) terminal asset prices. However, the smooth solution $V_{T, B}^{*}$ implies that the intersection with $V_{T, A}^{*}$ occurs at a higher asset price $S_{T}$ if compared to the intersection of $V_{T, C}^{*}$ and $V_{T, A}^{*}$. Let $s_{i, j}(i \neq j$, $i, j \in\{A, B, C\}$ ) denote the terminal asset price $S_{T}$ such that $V_{T, i}^{*}=V_{T, j}^{*}$. Equation (30) immediately gives

$$
V_{T, A}^{*}=V_{T, B}^{*} \Leftrightarrow V_{T, A}^{*}=V_{0} e^{r T}
$$

such that

$$
s_{A, B}=S_{0} e^{\left(r+\frac{1}{2}(m-1) \sigma^{2}\right) T} .
$$

With Equation (33), $\tilde{V}_{0} \leq V_{0}$ and $V_{0} \geq e^{-r T} G_{T}$ it follows

$$
s_{A, C}=S_{0} e^{\frac{1}{m}(g-r) T} e^{\left(r+\frac{1}{2}(m-1) \sigma^{2}\right) T}=e^{\frac{1}{m}(g-r) T} s_{A, B} \text { where } g:=\frac{1}{T} \ln \frac{G_{T}}{V_{0}} \leq r .
$$

Finally, one obtains

$$
s_{B, C}=S_{0} e^{\frac{1}{m}(\nu-r) T} e^{\left(r+\frac{1}{2}(m-1) \sigma^{2}\right) T}=e^{\frac{1}{m}(\nu-r) T} s_{A, B} \text { where } \nu:=\frac{1}{T} \ln \frac{G_{T}}{\tilde{V}_{0}-C_{0}} \geq r
$$

i.e. $s_{A, C} \leq s_{A, B} \leq s_{B, C}$. An illustration of the payoffs and the intersection points is given in Figure 1. ${ }^{14}$

We end this section by emphasizing one important consequence for the two protection mechanisms implied by the smooth and the kinked solutions, i.e. implied by the assumptions that marginal utility jumps gradually (respectively, discontinuously) to infinity. Notice that the smooth payoff $V_{T, B}^{*}$ implies that there is no probability mass on the event that the terminal value is equal to the guarantee $G_{T}$, i.e. $P\left(V_{T, B}^{*}=G_{T}\right)=0$. In contrast,

\footnotetext{
${ }^{14}$ If not mentioned otherwise, all illustration which are given in the following are based on parameters summarized in Table 2.
} 
for the kinked payoff $V_{T, C}^{*}$ it holds ${ }^{15}$

$$
P\left(V_{T, C}^{*}=G_{T}\right)=P\left(\phi\left(\tilde{V}_{0}, m^{*}\right) S_{T}^{m^{*}} \leq G_{T}\right)=P\left(\ln \frac{S_{T}}{S_{0}} \leq \ln \frac{\sqrt[m *]{\frac{G_{T}}{\phi\left(\tilde{V}_{0}, m^{*}\right)}}}{S_{0}}\right) .
$$

Using the definition of $\phi$, cf. Equation (12), and inserting $m^{*}=\frac{\mu-r}{\gamma \sigma^{2}}$ yields

$$
P\left(V_{T, C}^{*}=G_{T}\right)=1-\mathcal{N}\left(\frac{\ln \frac{\tilde{V}_{0}}{e^{-r T} G_{T}}-\frac{1}{2}\left(\frac{\lambda}{\gamma}\right)^{2} T}{\frac{\lambda}{\gamma} \sqrt{T}}+\lambda \sqrt{T}\right) \text { where } \lambda:=\frac{\mu-r}{\sigma} .
$$

\section{UtiLity LOSS CAUSED By GUARANTEES}

3.1. Justification of guarantees and empirical observations. There are a few comments necessary concerning the justification of guarantees. The optimization problems (B) and (C) are already based on an exogenously postulated guarantee such that one might doubt their capacity to give a meaningful justification of guarantees. However, there are some arguments which are in favor of a subsistence level. Similar reasonings are true with respect to optimization problem (C). In consequence, to some extent, guarantees can be explained with respect to the assumption that the investor's preferences can be described using the von Neumann and Morgenstern (1944) framework of expected utility. More recently, Doskeland and Nordahl (2008) justify the existence of guarantees through behavioral models. In particular, they use cumulative prospect theory as an example.

In the following, we do not give further justifications for the existence of guarantees or the popularity of portfolio insurance strategies but take them as given. However, we think in terms of utility losses caused by guarantees and compare the smooth and kinked payoff solutions. One possibility which is consistent with empirical observations is given by measuring the utility losses of guarantees with respect to a CRRA utility function where the parameter of risk aversion parameter is assumed to be above one $(\gamma>1) .{ }^{16}$

3.2. Utility loss. The performance of the strategy $\pi$ can be measured by its associated expected utility which can in turn be described by the certainty equivalent. It is defined as certain amount which makes the investor indifferent between achieving this certain

\footnotetext{
${ }^{15}$ Intuitively it is clear that a positive point mass on the event $\left\{V_{T}=G_{T}\right\}$ might indicate that the corresponding strategy is sensitive to the introduction of gap risk which is caused by asset price jumps. This problem is considered in the Sec. 4 where trading restrictions in the sense of discrete time trading and transaction costs are introduced.

${ }^{16} \mathrm{With}$ respect to the literature about the validity of CRRA utility functions and the parameter of risk aversion we refer to Munk (2008) and the literature given herein.
} 


\section{Loss rates}

\begin{tabular}{|l|l|}
\hline strategy $\pi$ & loss rate $l_{T, A}(\pi)$ \\
\hline$\pi_{t}^{C P}=m$ & $\frac{1}{2} \gamma \sigma^{2}\left(m^{*}-m\right)^{2}$ \\
$\pi_{t}^{C P P I}=m \frac{V_{t}-e^{-r(T-t)} G_{T}}{V_{t}}$ & $\frac{1}{(1-\gamma) T} \ln \left(\frac{\mathbb{E}_{P}\left[\left(\phi\left(V_{0}, m^{*}\right) S_{T}^{m^{*}}\right)^{1-\gamma}\right]}{\mathbb{E}_{P}\left[\left(G_{T}+\phi\left(V_{0}-e^{-r T} G_{T}, m\right) S_{T}^{m}\right)^{1-\gamma}\right]}\right)$ \\
$\pi_{t}^{O B P I}=\frac{\Delta^{P O}\left(t, S_{t} ; m, \frac{G_{T}}{\phi\left(\tilde{V}_{0}, m\right)}\right) S_{t}}{V_{t}}$ & $\frac{1}{(1-\gamma) T} \ln \left(\frac{\mathbb{E}_{P}\left[\left(\phi\left(V_{0}, m^{*}\right) S_{T}^{m *}\right)^{1-\gamma}\right]}{\mathbb{E}_{P}\left[\left(G_{T}+\left[\phi\left(\tilde{V}_{0}, m\right) S_{T}^{m}-G_{T}\right]^{+}\right)^{1-\gamma}\right]}\right)$ \\
\hline
\end{tabular}

TABLE 3. Loss rates with respect to $u_{A}(x)=\frac{x^{1-\gamma}}{1-\gamma}$.

amount (at $T$ ) or using the strategy $\pi$, i.e. the time $T$ certainty equivalent $C E_{T}$ of the strategy $\pi$ is defined by

$$
u\left(C E_{T}(\pi)\right)=\mathbb{E}_{P}\left[u\left(V_{T}(\pi)\right)\right] .
$$

Consider for example the utility function $u_{A}(x)=\frac{x^{1-\gamma}}{1-\gamma}$ and a CM strategy with investment proportion $m$, i.e.

$$
C E_{T, A}\left(\pi^{C M}\right)=\left(\mathbb{E}_{P}\left[\left(\phi\left(V_{0}, m\right) S_{T}^{m}\right)^{1-\gamma}\right]\right)^{\frac{1}{1-\gamma}}=\phi\left(V_{0}, m\right)\left(\mathbb{E}_{P}\left[S_{T}^{(1-\gamma) m}\right]\right)^{\frac{1}{1-\gamma}} .
$$

With $\mathbb{E}_{P}\left[S_{T}^{p}\right]=S_{0}^{p} \exp \left\{\left(p \mu-\frac{1}{2} p(1-p) \sigma^{2}\right) T\right\}$ it follows

$$
C E_{T, A}\left(\pi^{C M}\right)=\phi\left(V_{0}, m\right) S_{0}^{m} e^{\left(m \mu-\frac{1}{2} \gamma \sigma^{2} m(1-m(1-\gamma))\right) T}=V_{0} e^{\left(r+m(\mu-r)-\frac{1}{2} \gamma m^{2} \sigma^{2}\right) T} .
$$

Analogously, one obtains

$$
C E_{T, B}\left(\pi^{C P P I}\right)=G_{T}+\left(V_{0}-e^{-r T} G_{T}\right) e^{\left(r+m(\mu-r)-\frac{1}{2} \gamma m^{2} \sigma^{2}\right) T} .
$$

Thus, the certainty equivalent is lower for an investor with subsistence level than an investor without. We can also calculate the utility loss of an investor who follows a suboptimal strategy. It is described by the loss rate $l_{T, i}(\pi)$ of the strategy $\pi$ and the utility function $i(i \in\{A, B, C\})$ where

$$
l_{T, i}(\pi):=\frac{\ln \left(\frac{C E_{T, i}^{*}}{C E_{T, i}(\pi)}\right)}{T} .
$$

$C E_{T, i}^{*}$ denotes the certainty equivalent of the optimal strategy $\pi_{i}^{*}=\left(\pi_{t, i}^{*}\right)_{0 \leq t \leq T}$ while $C E_{T, i}(\pi)$ the one of the suboptimal strategy $\pi=\left(\pi_{t}\right)_{0 \leq t \leq T}$.

The loss rates with respect to the utility function $u_{A}(x)=\frac{x^{1-\gamma}}{1-\gamma}$ are summarized in Table 3 for CM, CPPI and OBPI strategies with strategy parameter $m .{ }^{17}$ Notice that a loss rate which is higher than the one of a risk-free investment, i.e. $\pi_{t}^{C M}=0$, implies that

\footnotetext{
${ }^{17}$ If not mentioned otherwise, we refer to $u_{A}$.
} 
Loss rates depending on $m$
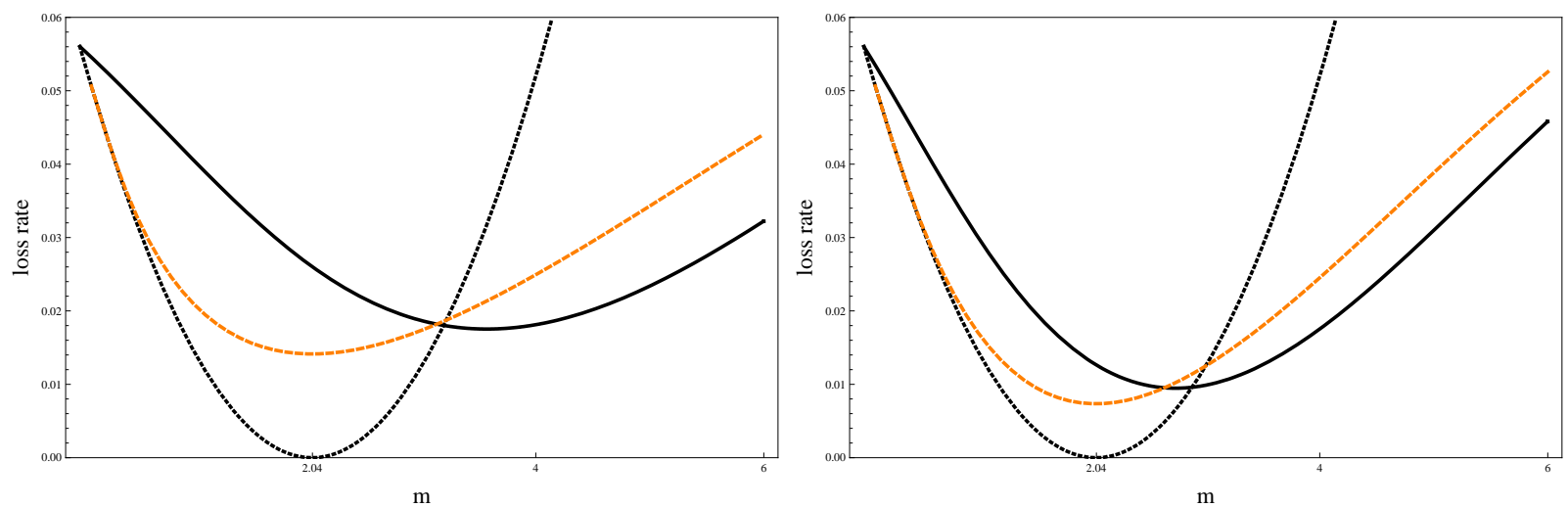

Figure 2. Loss rates w.r.t. $u=u_{A}$ for CPPI (solid lines), OBPI (dashed) and CM (dotted) strategies with varying parameter $m$. The parameters are given in Table 2. The investment horizon is $T=10$ years (left graph) and $T=20$ years (right graph).

the associated strategy is prohibitively bad. This critical loss rate is equal to $\frac{1}{2} \gamma\left(\sigma m^{*}\right)^{2}$, cf. Table $3 .{ }^{18}$ Notice that for $m=m^{*}$ it holds ${ }^{19}$

$$
l_{T, A}\left(\pi^{C P}\right)=0<l_{T, A}\left(\pi^{O B P I}\right)<l_{T, A}\left(\pi^{C P P I}\right) .
$$

For $m=m^{*}$, the utility loss implied by the guarantee is higher for the CPPI than for of the OBPI. This is illustrated in Figure 2. In addition, notice that the loss rates of the $\mathrm{CM}$ strategies are symmetric in the sense that for $\epsilon>0$, a strategy parameter $m=m^{*}+\epsilon$ implies the same loss rate as the parameter $m=m^{*}-\epsilon$. In contrast, portfolio insurance strategies yield a lower loss rates in the case of $m=m^{*}+\epsilon$ than for $m=m^{*}-\epsilon$. Intuitively, this is clear since the protection feature implies that the portfolio weights of the portfolio insurance strategies are too low compared to the optimal investment proportion $\mathrm{m}^{*}$, cf. Inequalities (19) and (28).

For the comparison of CPPI and OBPI, it is important to keep in mind that $m^{*}$ is the optimal OBPI parameter for $u=u_{A}$. In contrast, $m^{*}$ is not the optimal parameter for $u=u_{A}$, but for $u=u_{B}$ which includes a subsistence level. In order to compare the loss rates implied by CPPI and OBPI w.r.t. $u=u_{A}$, it is thus necessary to consider the maximization problem

$$
\max _{\pi \in \Pi^{C P P I}} \mathbb{E}_{P}\left[u_{A}\left(V_{T}(\pi)\right)\right]=\max _{m} \mathbb{E}_{P}\left[\frac{\left(G_{T}+\phi\left(V_{0}-e^{-r T} G_{T}, m\right) S_{T}^{m}\right)^{1-\gamma}}{1-\gamma}\right] .
$$

\footnotetext{
${ }^{18}$ For the basic parameter constellation it holds $\frac{1}{2} \gamma\left(\sigma m^{*}\right)^{2}=0.056$.

${ }^{19}$ For $m=m^{*}$, the inequality $l_{T, A}\left(\pi^{O B P I}\right)<l_{T, A}\left(\pi^{C P P I}\right)$ is obvious since $\pi^{C P P I}$ is suboptimal w.r.t. the optimization problem $(\mathrm{C})$ where $u_{C}=u_{A}$ and $\pi^{O B P I}$ is optimal.
} 
Loss rates depending on $m$
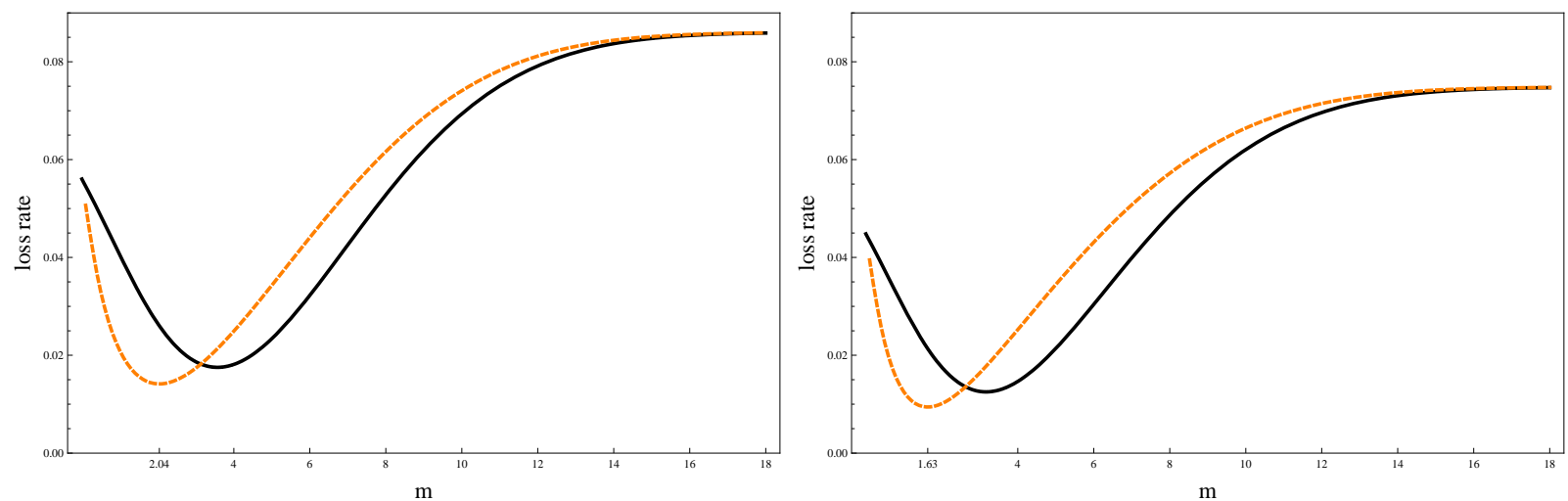

Figure 3. Loss rates implied by CPPI (solid lines) and OBPI (dashed lines) for varying $m$, cf. Table 3 . The risk aversion is $\gamma=1.2$ (left figure) and $\gamma=1.5$ (right figure), for the other parameters, cf. Table 2.

\section{Minimal loss rates}

\begin{tabular}{|c|c||l|l|l|l|l|}
\hline strategy & $\gamma \backslash T$ & 1 & 2 & 5 & 10 & 20 \\
\hline \hline CPPI & 1.2 & $0.040(11.32)$ & $0.035(7.83)$ & $0.026(4.91)$ & $0.018(3.57)$ & $0.010(2.73)$ \\
OBPI & 1.2 & $0.037(2.04)$ & $0.031(2.04)$ & $0.022(2.04)$ & $0.014(2.04)$ & $0.007(2.04)$ \\
\hline \hline CPPI & 1.5 & $0.031(10.60)$ & $0.026(7.25)$ & $0.019(4.45)$ & $0.013(3.16)$ & $0.007(2.36)$ \\
OBPI & 1.5 & $0.028(1.63)$ & $0.023(1.63)$ & $0.015(1.63)$ & $0.009(1.63)$ & $0.005(1.63)$ \\
\hline \hline CPPI & 1.8 & $0.024(10.03)$ & $0.020(6.80)$ & $0.014(4.10)$ & $0.009(2.86)$ & $0.005(2.08)$ \\
OBPI & 1.8 & $0.021(1.34)$ & $0.017(1.34)$ & $0.011(1.34)$ & $0.007(1.34)$ & $0.003(1.34)$ \\
\hline \hline
\end{tabular}

TABLE 4. Minimal loss rates $\left(u_{A}\right.$-optimal strategy parameter $m$ ) for varying $T$ and $\gamma$ where the other parameters are given as in Table 2.

Figure 3 illustrates the loss rates for CPPI and OBPI strategies with varying parameter $m$. In addition, the minimal loss rates are summarized in Table 4, i.e. the loss rates for OBPI strategies with $m=m^{*}$ and CPPI for $m=m^{* *}$. Although the OBPI strategy with parameter $m=m^{*}$ is, per construction, the $u_{A}$-utility maximizing portfolio insurance strategy, the additional loss of the CPPI strategy with parameter $m=m^{* *}$ which is measured by the difference of its loss rate and the one of the OBPI is rather low. Intuitively, this is explained as follows...

\section{UTILITY LOSS CAUSED BY TRADING RESTRICTIONS AND TRANSACTION COSTS}

It is important to notice that in practice the concept of portfolio insurance is impeded by market frictions. The protection mechanism of portfolio insurance implies that the 
asset exposure is to be reduced when the asset price decreases. A sudden drop in the asset price where the investor is not able to adjust his portfolio adequately, causes a gap risk, i.e. the risk that the terminal guarantee is not achieved. One illustrative and meaningful approach to capture gap risk is given by the introduction of trading restrictions in the sense of discrete-time trading and transaction costs. ${ }^{20}$ In particular, we consider transaction costs which are proportional to a change in the position of the risky asset. The proportionality factor is denoted by $\theta$. As before, we assume that the present value of the terminal guarantee $G_{T}$ prevailing at $T$ is lower than the initial investment $V_{0}$, i.e. $V_{0}>e^{-r T} G_{T}$.

Let $\tau^{n}$ denote a sequence of equidistant refinements of the interval $[0, T]$, i.e. $\tau^{n}=$ $\left\{t_{0}^{n}=0<t_{1}^{n}<\cdots<t_{n-1}^{n}<t_{n}^{n}=T\right\}$, where $t_{k+1}^{n}-t_{k}^{n}=\frac{T}{n}$ for $k=0, \cdots, n-1$. To simplify the notation, we drop the superscript $n$ and denote the set of trading dates with $\tau$ instead of $\tau^{n}$. The restriction that trading is only possible immediately after $t_{k} \in \tau$ implies that the number of shares held in the risky asset is constant over the intervals ]$\left.t_{i}, t_{i+1}\right]$ for $i=0, \ldots, n-1$. However, the fractions of wealth which are invested in the assets change as assets prices fluctuate. Thus, it is necessary to consider the number $\rho_{S}$ of shares held in the risky asset and the number $\rho_{B}$ of zero bonds with maturity $T$, i.e. the tupel $\rho=\left(\rho_{S}, \rho_{B}\right)$.

4.1. Discrete-time CPPI. Along the lines of Balder et al. (2009), we consider a discretetime CPPI version $\rho^{\tau}=\left(\rho_{S}^{\tau}, \rho_{B}^{\tau}\right)$ which is for $\left.\left.t \in\right] t_{k}, t_{k+1}\right]$ and $k=0, \ldots, n-1$ defined by

$$
\rho_{t, S}^{\tau}:=\max \left\{\frac{m C_{t_{k}}^{\tau}}{S_{t_{k}}}, 0\right\}, \quad \rho_{t, B}^{\tau}:=\frac{1}{B_{t_{k}}}\left(V_{t_{k}}^{\tau}-\rho_{t, S}^{\tau} S_{t_{k}}\right)
$$

Notice that we do not allow for short positions in the risky asset, i.e. the asset exposure is bounded below by zero. However, similar as for the simple (continuous-time) CPPI, the discrete-time CPPI does not include short sale restrictions on the riskless asset.The terminal value of the discrete-time CPPI is given by

$$
\begin{aligned}
V_{T}^{\tau} & =G_{T}+\left(V_{t_{0}}-e^{-r T} G_{T}\right) \prod_{i=1}^{\min \{s, n\}} m\left(\frac{S_{t_{i}}}{S_{t_{i-1}}}-K(m)\right) e^{r\left(T-\min \left\{t_{s}, T\right\}\right)} \\
\text { where } K(m) & :=\frac{m-1}{m} e^{r \frac{T}{n}}
\end{aligned}
$$

\footnotetext{
${ }^{20}$ Notice that discrete-time trading is one possibility to introduce asset price jumps. In addition, it also allows to take into account for transaction costs which are of practical importance if one decides wether to use the CPPI or OBPI protection mechanism.
} 
and where $t_{s}:=\min \left\{t_{k} \in \tau \mid V_{t_{k}}<e^{-r\left(T-t_{k}\right)} G_{T}\right\}$ and $t_{s}=\infty$ if the minimum is not attained. In particular, it holds that $t_{s}:=\min \left\{t_{k} \in \tau \mid \frac{S_{t_{k}}}{S_{t_{k-1}}}<K(m)\right\}$. For $m>1$, the value of the simple CPPI can drop below the floor. Therefore, the discrete-time CPPI version introduces a gap risk, i.e. a risk that the guarantee is not honored. ${ }^{21}$

Besides discrete-time trading, we take also transaction costs into account. Along the lines of Black and Perold (1992) we assume that the transaction costs are financed by a reduction of the asset exposure arising in the case without transaction costs, i.e. ${ }^{22}$ the discretetime CPPI version with transaction costs $\rho^{\tau, T A}=\left(\rho_{S}^{\tau, T A}, \rho_{B}^{\tau, T A}\right)$ is, for $\left.\left.t \in\right] t_{k}, t_{k+1}\right]$ and $k=0, \ldots, n-1$, defined by

$$
\rho_{t, S}^{\tau, T A}:=\max \left\{\frac{m C_{t_{k^{+}}}^{\tau, T A}}{S_{t_{k}}}, 0\right\}, \rho_{t, B}^{\tau}:=\frac{1}{B_{t_{k}}}\left(V_{t_{k^{+}}}^{\tau, T A}-\rho_{t, S}^{\tau, T A} S_{t_{k}}\right) .
$$

where $V_{t_{k^{+}}}^{\tau, T A}\left(C_{t_{k^{+}}}^{\tau, T A}:=V_{t_{k^{+}}}^{\tau, T A}-e^{-r\left(T-t_{k}\right)} G_{T}\right)$ denotes the portfolio (cushion) value immediately after $t_{k}$, i.e. the value net of transaction costs which are proportional to the asset price $S_{t_{k}}$. First, consider the portfolio value $V_{t_{k}}^{\tau, T A}$ before transaction costs, i.e. $V_{t_{0}}^{\tau, T A}:=V_{t_{0}}$ and for $k=1, \ldots, n$

$$
\begin{aligned}
V_{t_{k}}^{\tau, T A} & :=\rho_{t_{k}, S}^{\tau, T A} S_{t_{k}}+\rho_{t_{k}, B}^{\tau, T A} B_{t_{k}} \\
& =\max \left\{\frac{m C_{t_{k-1}+}^{\tau, T A}}{S_{t_{k-1}}}, 0\right\} S_{t_{k}}+\left(V_{t_{k-1}+}^{\tau, T A}-\rho_{t_{k}, S}^{\tau, T A} S_{t_{k-1}}\right) e^{r\left(t_{k}-t_{k-1}\right)} \\
& =m \max \left\{C_{t_{k-1}+}^{\tau, T A}, 0\right\}\left(\frac{S_{t_{k}}}{S_{t_{k-1}}}-e^{r\left(t_{k}-t_{k-1}\right)}\right)+V_{t_{k-1}+}^{\tau, T A} e^{r\left(t_{k}-t_{k-1}\right)}
\end{aligned}
$$

Consider now the adjustment to the proportional transaction costs which are due immediately after the trading dates. Assuming that the transaction costs are also due at $t_{0}$ and that the asset positions are transferred into a cash position immediately after $t_{n}$ is

\footnotetext{
${ }^{21}$ For a detailed analysis of the gap we refer to Balder et al. (2009).

${ }^{22}$ This can be justified by the argument that the protection feature of the CPPI is based on a prespecified riskfree investment such that the introduction of transaction costs must not change the number of risk free bonds which are prescribed by the CPPI method (without transaction costs).
} 
consistent to the following definitions

$$
\begin{aligned}
V_{t_{0^{+}}}^{\tau, T A} & :=V_{t_{0}}-\rho_{S, t_{0^{+}}}^{\tau, T A} \theta S_{t_{0}}=V_{t_{0}}-m \theta C_{t_{0^{+}}}^{\tau, T A} \\
V_{t_{k^{+}}}^{\tau, T A} & :=V_{t_{k}}^{\tau, T A}-\left|\rho_{t_{k^{+}, S}^{\tau, T A}}-\rho_{t_{k}, S}^{\tau, T A}\right| \theta S_{t_{k}} \\
& =V_{t_{k}}^{\tau, T A}-m \theta\left|\max \left\{C_{t_{k^{+}}}^{\tau, T A}, 0\right\}-\max \left\{C_{t_{k_{-}+}+, T A}^{\tau,}, 0\right\} \frac{S_{t_{k}}}{S_{t_{k-1}}}\right| \\
V_{t_{n}+}^{\tau, T A} & :=V_{t_{n}}^{\tau, T A}-\rho_{t_{n}, S}^{\tau, T A} \theta S_{t_{n}} \\
& =V_{t_{n}}^{\tau, T A}-m \theta \max \left\{C_{t_{n-1^{+}}}^{\tau, T A}, 0\right\} \frac{S_{t_{n}}}{S_{t_{n-1}}} .
\end{aligned}
$$

With Equation (47), it immediately follows $C_{t_{0}+}:=\frac{1}{1+\theta m} C_{t_{0}}$. Using Equation (48) and Equation (46) imply that for for $C_{t_{k}+}>0(k=0, \ldots n-1)$ and $\theta<\frac{1}{m}$ it holds ${ }^{23}$

$$
\begin{aligned}
C_{t_{k+1}+} & =C_{t_{k+1}}-m \theta\left|\max \left\{C_{t_{k+1}+}, 0\right\}-C_{t_{k}+} \frac{S_{t_{k+1}}}{S_{t_{k}}}\right| \\
& =\left\{\begin{array}{lr}
C_{t_{k}+}\left(\frac{1+\theta}{1+\theta m} m \frac{S_{t_{k+1}}}{S_{t_{k}}}-\frac{m-1}{1+\theta m} e^{r \frac{T}{n}}\right) & \text { for } e^{r \frac{T}{n}} \leq \frac{S_{t_{k+1}}}{S_{t_{k}}} \\
C_{t_{k}+}\left(\frac{1-\theta}{1-\theta m} m \frac{S_{t_{k+1}}}{S_{t_{k}}}-\frac{m-1}{1-\theta m} e^{r \frac{T}{n}}\right) & \text { for } \frac{m-1}{m(1-\theta)} e^{r \frac{T}{n}} \leq \frac{S_{t_{k+1}}}{S_{t_{k}}}<e^{r \frac{T}{n}} \\
C_{t_{k}+}\left((1-\theta) m \frac{S_{t_{k+1}}}{S_{t_{k}}}-(m-1) e^{r \frac{T}{n}}\right) & \text { for } \frac{S_{t_{k+1}}}{S_{t_{k}}}<\frac{m-1}{m(1-\theta)} e^{r \frac{T}{n}}
\end{array}\right.
\end{aligned}
$$

For $C_{t_{k}+} \leq 0$ it follows $C_{t_{k+1}+}=C_{t_{k+1}}=e^{r \frac{T}{n}} C_{t_{k}+}$. It is worth mentioning that the event $\left\{V_{t_{n+}}^{\tau, T A}<G_{T}\right\}$ corresponds to the event that the adjusted cushion drops below zero during the investment horizon. Notice that for, $C_{t_{k}+}>0$

$$
\left\{C_{t_{k+1}+}<0\right\} \Leftrightarrow\left\{\frac{S_{t_{k+1}}}{S_{t_{k}}}<\frac{m-1}{m(1-\theta)} e^{r \frac{T}{n}}\right\}=: A_{k+1}
$$

Since the complementary of the event $\left\{\cup_{k=0}^{n-1} A_{k+1}\right\}$ is given by the event that all asset price increments are above $\frac{m-1}{m(1-\theta)} e^{r \frac{T}{n}}$ it follows with the assumption that the asset price increments are independent and identically distributed that ${ }^{24}$

$$
\begin{aligned}
P\left(V_{t_{n}}^{\tau, T A}<G_{T}\right) & =1-\left(P\left(\frac{S_{t_{1}}}{S_{t_{0}}}>\frac{m-1}{m(1-\theta)} e^{r \frac{T}{n}}\right)\right)^{n} \\
& =1-\left(\mathcal{N}\left(d_{2}^{\mathrm{TA}}(\theta)\right)\right)^{n} \\
\text { where } d_{2}^{\mathrm{TA}}(\theta) & :=\frac{\ln \frac{(1-\theta) m}{m-1}+(\mu-r) \frac{T}{n}-\frac{1}{2} \sigma^{2} \frac{T}{n}}{\sigma \sqrt{\frac{T}{n}}}
\end{aligned}
$$

\footnotetext{
${ }^{23}$ Notice that $\frac{m-1}{m(1-\theta)} e^{r \frac{T}{n}}<e^{r \frac{T}{n}} \Leftrightarrow \theta<\frac{1}{m}$.

${ }^{24}$ In addition to the shortfall probability, the simple model setup also allows a closed-form calculation of other risk measures such as the expected shortfall, cf. Balder et al. (2009).
} 
Loss rates depending on $m$
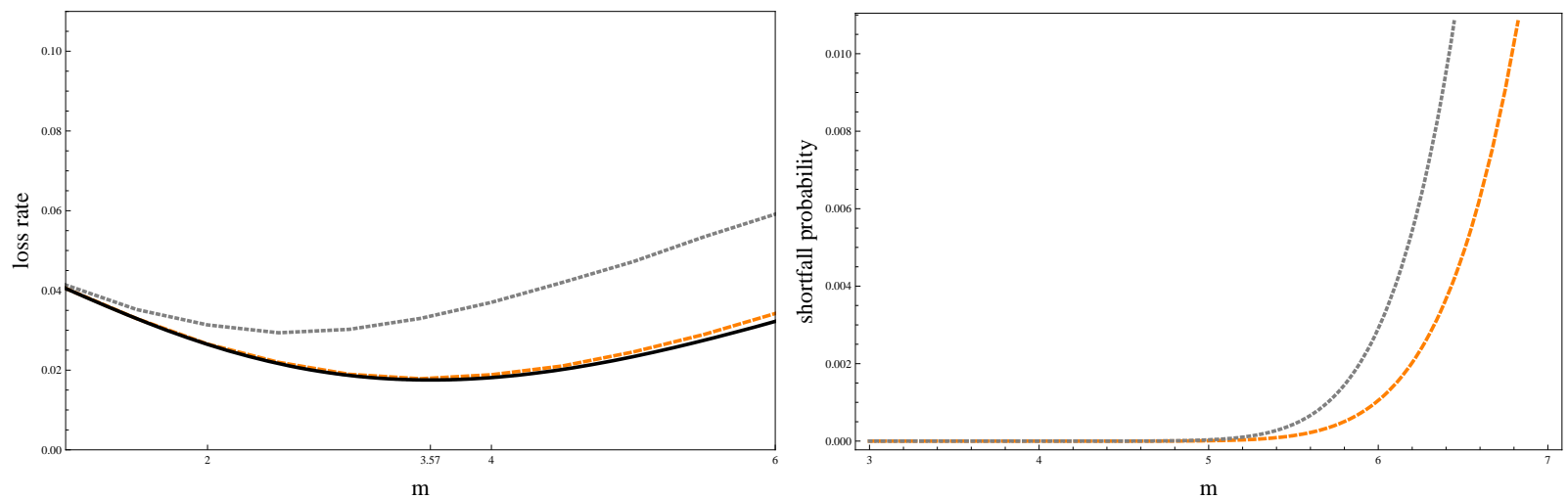

FiguRE 4. Loss rates w.r.t. $u=u_{A}$ (shortfall probabilities) implied by continuous-time CPPI (solid line), monthly CPPI without transaction costs (dashed lines) and monthly CPPI with $\theta=0.01$ (dotted line) for varying $m$. The parameter setup is given in Table 2 .

4.2. Discrete-time option based strategy. Recall that according to Equation (26) the (continuous-time) self-financing and duplicating strategy for the $T$-payoff $G_{T}+$ $\phi\left(\tilde{V}_{0}, m\right)\left[S_{T}^{m}-\frac{G_{T}}{\phi\left(\tilde{V}_{0}, m\right)}\right]^{+}$is given by

$$
\begin{aligned}
\rho_{t, S} & :=\phi\left(\tilde{V}_{0}, m\right) \Delta^{P O}\left(t, S_{t} ; m, \frac{G_{T}}{\phi\left(\tilde{V}_{0}, m\right)}\right) \\
\rho_{t, B} & :=G_{T}+\frac{\phi\left(\tilde{V}_{0}, m\right) P O\left(t, S_{t} ; m, \frac{G_{T}}{\phi\left(\tilde{V}_{0}, m\right)}\right)-\rho_{t, S} S_{t}}{B(t, T)}
\end{aligned}
$$

where $\Delta^{P O}$ is defined as in Equation (27). We consider as a discrete-time version of an arbitrary continuous-time trading strategy $\rho^{\tau}=\left(\rho_{S}^{\tau}, \rho_{B}^{\tau}\right)$ with respect to the trading dates $\tau$

$$
\left.\left.\rho_{t}^{\tau}:=\rho_{t_{k}^{n}} \text { for } t \in\right] t_{k}, t_{k+1}\right] \text { and for all } t \in[0, T] .
$$

Setting $V_{0}(\rho ; \tau):=V_{0}(\rho)$, the value process $V(\rho ; \tau)$ which is associated with $\rho^{\tau}$ is

$$
\left.\left.V_{t}(\rho ; \tau)=\rho_{t_{k}, S} S_{t}+\rho_{t_{k}, B} e^{-r(T-t)} \text { for } t \in\right] t_{k}, t_{k+1}\right] \text { and } 0 \leq k \leq n-1
$$

Notice that, in general, the discrete-time version of a continuous-time strategy is not self-financing. In particular, there are in- or out-flows from the portfolio which occur immediately after a trading date $t_{k+1}(k=0, \ldots, n-1)$. Formally, the costs of discretization $\xi_{t_{k+1}}^{\text {dis }}(\rho ; \tau)$ which occur immediately after the trading date $t_{k+1}$ are defined by

$$
\begin{aligned}
\xi_{t_{k+1}}^{\text {dis }}(\rho ; \tau) & :=V_{t_{k+1}}(\rho)-V_{t_{k+1}}(\rho ; \tau) \\
& =\left(\rho_{t_{k+1}, S}-\rho_{t_{k}, S}\right) S_{t_{k+1}}+\left(\rho_{t_{k+1}, B}-\rho_{t_{k}, B}\right) e^{-r\left(T-t_{k+1}\right)}
\end{aligned}
$$


Loss rates depending on $m$
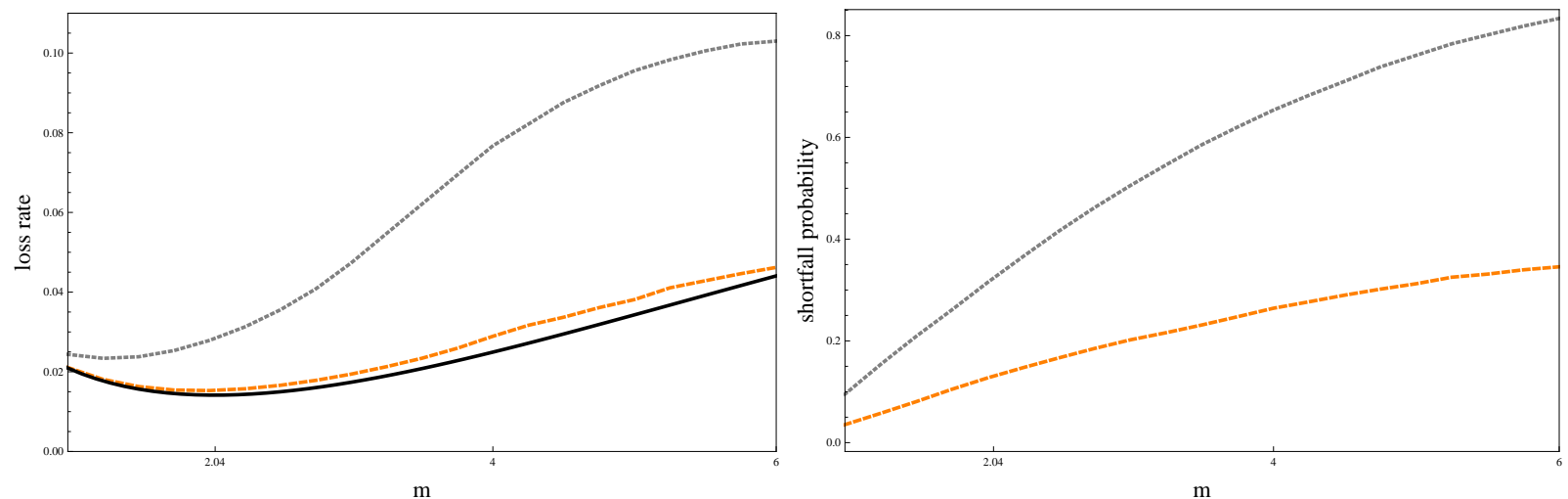

FiguRE 5. Loss rates w.r.t. $u=u_{A}$ (shortfall probabilities) in the Merton setup implied by continuous-time OBPI (solid line), monthly OBPI without transaction costs (dashed lines) and monthly OBPI with $\theta=0.01$ (dotted line) for varying $m$. The parameter setup is given in Table 2 .

Notice that negative costs refer to inflows while positive costs imply that further money is needed to continue the strategy.

Taking into account for proportional transaction costs also implies that there are transaction $\operatorname{costs} \xi_{t_{k+1}}^{T A}(\rho ; \tau)$ which occur immediately after a trading date $t_{k+1}$, i.e.

$$
\begin{aligned}
\xi_{t_{0}}^{T A}(\rho ; \tau) & :=\left|\rho_{t_{0}, S}\right| \theta S_{t_{0}} \\
\xi_{t_{k+1}}^{T A}(\rho ; \tau) & =\left|\rho_{t_{k+1}, S}-\rho_{t_{k}, S}\right| \theta S_{t_{k+1}} \text { for } k=0, \ldots, n-2 \\
\text { and } \xi_{t_{n}}^{T A}(\rho ; \tau) & :=\left|\rho_{t_{n-1}, S}\right| \theta S_{t_{n}} .
\end{aligned}
$$

Defining the payoff $\bar{V}_{T}(\rho ; \tau)$ according to the assumption that the inflows into the strategy are lent according to the interest rate $r$ and outflows are saved according to $r$ gives

$$
\bar{V}_{T}(\rho ; \tau):=V_{t_{n}}(\rho)-\left[\xi_{t_{0}}^{T A} e^{r\left(T-t_{0}\right)}+\sum_{k=0}^{n-1}\left(\xi_{t_{k+1}}^{\text {dis }}(\rho ; \tau)+\xi_{t_{k+1}}^{T A}(\rho ; \tau)\right) e^{r\left(t_{n}-t_{k+1}\right)}\right]
$$

4.3. Comments on utility loss and shortfall probability. The loss rates w.r.t. $u=u_{A}$ (shortfall probabilities) of the above discrete-time versions are illustrated in Figure 4 and Figure $5 .^{25}$ Observe, that for both strategies, OBPI and CPPI, the loss which is in the first instance caused by time-discretizing the strategies is rather low. However, there is a huge impact caused by transaction costs where the effect is even more

\footnotetext{
${ }^{25}$ Notice that the introduction of gap risk. i.e. a strictly positive shortfall probability, gives a loss of minus infinity in the case of $u=u_{B}$ and $u=u_{C}$.
} 
Distribution of discrete OBPI (CPPI) for $m=m^{*}\left(m=m^{* *}\right)$
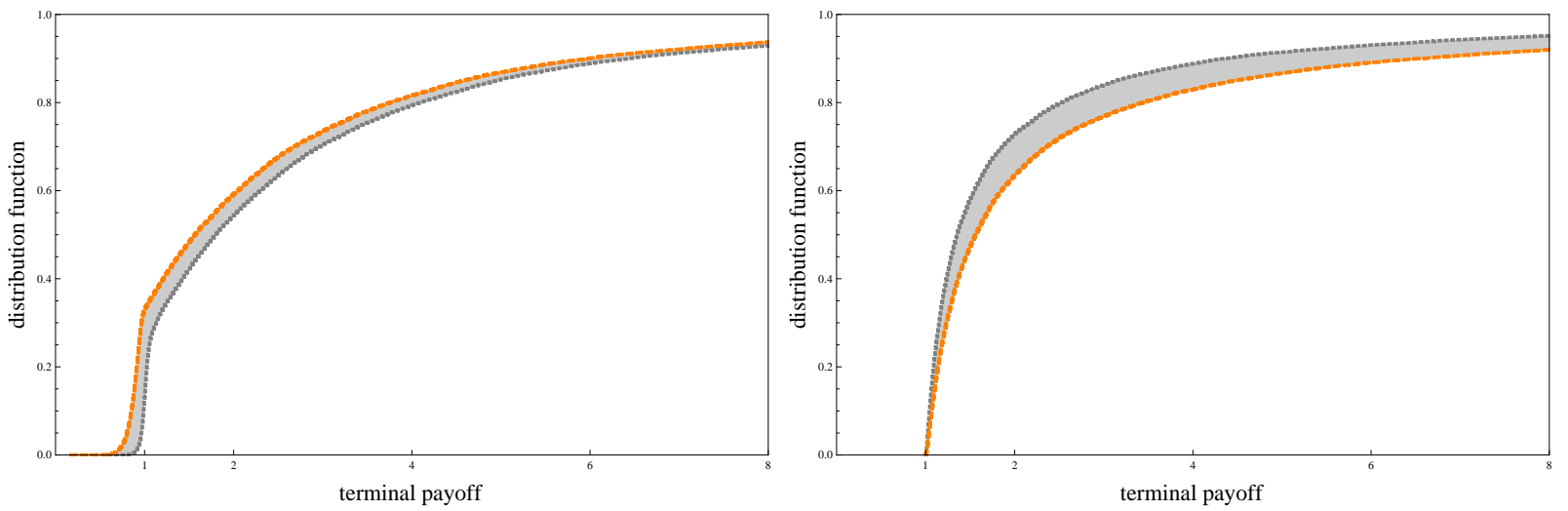

Figure 6. Distribution functions of terminal values for $\theta=0(\theta=0.01$, respectively).

pronounced for the OBPI than for the CPPI method. ${ }^{26}$ Recall that loss rates above the one of a risk-free investment are prohibitive. In the case of the basic parameter constellation, this critical value w.r.t. $u=u_{A}$ is equal to $\frac{1}{2} \gamma(\sigma m)^{2}=0.056$. In particular, if the strategy parameter $m$ is not chosen in a cautious way, portfolio insurance can get prohibitively bad because of transaction costs.

Consider now the shortfall probability. Recall that, in contrast to the continuous-time CPPI strategy, the continuous-time OBPI results in a strictly positive probability that the payoff is not above the terminal guarantee. Thus, it is to be expected that the shortfall probability of the OBPI is more sensitive to discrete-time trading and transaction costs than the one of the CPPI, cf. the right graphs presented in Figure 4 and Figure 5. This effect is also illustrated in Figure 6 where the distribution function of the discrete-time versions of OBPI with $m=m^{*}$ and CPPI with $m=m^{* *}$. However, to some extend the sensitivity of the OBPI to the gap risk measured by the shortfall probability is also caused by the dicretization scheme. While the transaction costs are financed via the cushion, this is not true in the case of the discrete-time OBPI.

\section{Utility LOSS CAUSED BY GUARANTEES AND BORROWING CONSTRAINTS}

To be practically relevant, it is necessary to take borrowing constraints into account .Notice that the basic CPPI approach can result in arbitrarily high investment proportions.

\footnotetext{
${ }^{26}$ Intuitively, it is clear that in the limit to continuous-time trading, the transaction costs eat away the cushion which allows a risky investment. The convergence of the value (cushion) process of the CPPI is for example analyzed in Balder et al. (2009).
} 
Incorporating borrowing constraints into the classic CPPI strategy straightforwardly results in the capped CPPI which we abbreviate with CCP. ${ }^{27}$ The capped CPPI strategy $\rho^{C C P}=\left(\rho_{S}^{\mathrm{CCP}}, \rho_{B}^{\mathrm{CCP}}\right)$ is defined by

$$
\begin{aligned}
\rho_{t, S}^{\mathrm{CCP}}=\frac{\min \left(\omega V_{t}^{\mathrm{CCP}}, m C_{t}^{\mathrm{CCP}}\right)}{S_{t}}, \quad \rho_{t, B}^{\mathrm{CCP}} & =\frac{V_{t}^{\mathrm{CCP}}-\rho_{t, S}^{\mathrm{CCP}} S_{t}}{B_{t}} \\
\text { where } C_{t}^{\mathrm{CCP}} & :=V_{t}^{\mathrm{CCP}}-e^{-r(T-t)} G_{T} .
\end{aligned}
$$

and where $w(w \geq 1)$ denotes the restriction on the investment proportion. In the following, we refer to borrowing constraints in the strict sense, i.e. we assume that $\omega$ is set equal to one.

It is worth mentioning that the borrowing constraints introduce a path-dependence and the payoff implied by the capped CPPI version can not be stated as a function of the terminal asset price as it is the case without borrowing constraints. Intuitively, it is to be expected that the path dependence yields an additional utility loss in a Black/Scholes type model setup. In order to calculate the loss rate, we consider the distribution of the terminal value of the CP strategy. Let $f$ denote the density function of the terminal value of the capped CPPI. Then it holds

$$
\begin{aligned}
\mathbb{E}\left[u_{A}\left(\pi^{C C P}\right)\right] & =\int_{G_{T}}^{\infty} u_{A}(v) f(v) d v \\
C E_{T, A}\left(\pi^{C C P}\right) & =\left(\int_{G_{T}}^{\infty} v^{1-\gamma} f(v) d v\right)^{\frac{1}{1-\gamma}} \\
\text { such that } l_{T, A}\left(\pi^{C C P}\right) & =\frac{1}{T} \ln \left(\frac{V_{0} e^{\left(r+m^{+}(\mu-r)-\frac{1}{2} \gamma\left(m^{*} \sigma\right)^{2}\right) T}}{\left(\int_{G_{T}}^{\infty} v^{1-\gamma} f(v) d v\right)^{\frac{1}{1-\gamma}}}\right) .
\end{aligned}
$$

For details on the distribution of the capped CPPI we refer to Balder and Mahayni (2008). Basically, the distribution can be obtained by considering the process $(X)_{0 \leq t \leq T}$ which is given by the dynamics

$$
d X_{t}=\Theta\left(X_{t}\right) d t+d W_{t}
$$

where

$$
\Theta(x)=\left\{\begin{array}{ll}
\frac{\mu-r}{\sigma}-\frac{1}{2} m \sigma & x \leq 0 \\
\frac{\mu-r}{\sigma}-\frac{1}{2} \sigma & x>0
\end{array} \text { and } X_{0}= \begin{cases}\frac{1}{\sigma} \ln \frac{(m-1) V_{0}}{m G_{0}} & m C_{0} \geq V_{0} \\
\frac{1}{m \sigma} \ln \frac{(m-1) C_{0}}{G_{0}} & m C_{0}<V_{0}\end{cases}\right.
$$

\footnotetext{
${ }^{27}$ This is also true in the case of the OBPI strategy which is based on synthesizing a power option with a power $p>1$. However, it is not straightforward how to incorporate borrowing constraints for the OBPI. One possibility is given by setting $m=1$, i.e. referring to a standard option instead of a power option. This is also done in practice but not considered in the following.
} 
Loss rates depending on $m$
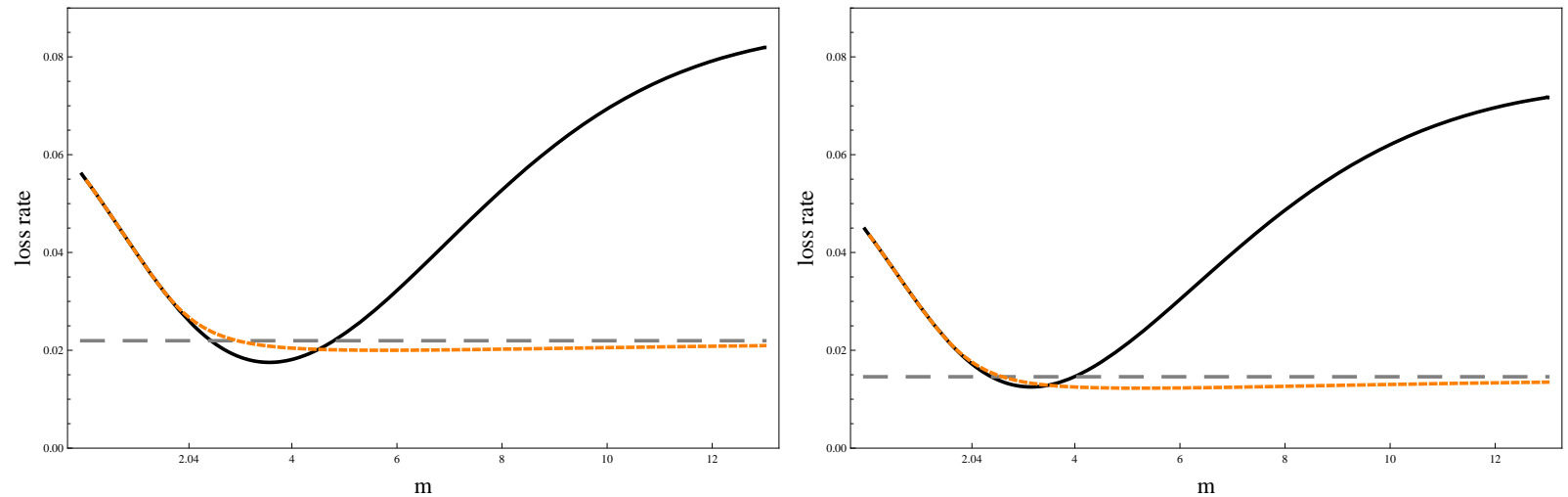

Figure 7. Loss rates w.r.t. $u=u_{A}$ implied by CPPI (solid lines) and capped CPPI (dashed lines) for varying $m$. The risk aversion is $\gamma=1.2$ (left figure) and $\gamma=1.5$ (right figure), the other parameters are as in Table 2. The constant line gives the loss rate of a stop-loss strategy.

Along the lines of Balder and Mahayni (2008), one can show that the value process $\left(V_{t}^{\mathrm{CCP}}\right)_{0 \leq t \leq T}$ and cushion process $\left(C_{t}^{\mathrm{CCP}}\right)_{0 \leq t \leq T}$ are, for $\omega=1$, given by

$$
V_{t}^{\mathrm{CCP}}=G_{t} \begin{cases}\frac{m}{m-1} e^{\sigma X_{t}} & X_{t} \geq 0 \\ \left(1+\frac{1}{m-1} e^{\sigma m X_{t}}\right) & X_{t}<0\end{cases}
$$

and

$$
C_{t}^{\mathrm{CCP}}=G_{t}\left\{\begin{array}{ll}
\left(\frac{m}{m-1} e^{\sigma X_{t}}-1\right) & X_{t} \geq 0 \\
\frac{1}{m-1} e^{m \sigma X_{t}} & X_{t}<0
\end{array} .\right.
$$

In particular, it holds

$$
P\left[V_{t}^{\mathrm{CCP}} \in d v\right]= \begin{cases}\frac{1}{\sigma v} p\left(\frac{\ln \frac{(m-1) v}{m G_{t}}}{\sigma}\right) d v & v \geq \frac{m}{m-1} G_{t} \\ \frac{1}{\sigma m\left(v-G_{t}\right)} p\left(\frac{\left.\ln \frac{(m-1)\left(v-G_{t}\right)}{G_{t}}\right)}{\sigma m}\right) d v & v<\frac{m}{m-1} G_{t}\end{cases}
$$

where $p(x):=P_{X_{0}}\left(X_{t} \in d x\right)$.

The loss rate implied by the capped CPPI is illustrated in Figure 7 . Notice that for $m \rightarrow \infty$, the capped CPPI converges to the stop-loss strategy, cf. Black and Perold (1992). Obviously, the loss rate converges, too. It is interesting to observe that the loss-rate of the capped CPPI is smaller than the loss-rate of the stop-loss-strategy for $m \geq m^{* *}$. 


\section{Conclusion}

The popularity of portfolio insurance strategies including a strictly positive guarantee component with respect to a fixed investment horizon can be explained by various reasons like regulatory requirements or behavioral finance models. To some extend, the justification of positive guarantees is also possible assuming that the investor's preferences can be described using the von Neumann Morgenstern framework of expected utility.

Dating back to Merton, it is well known that in a Black/Scholes model setup and for a CRRA utility function, the optimal strategy is to invest a constant fraction of wealth into the risky asset. Such a constant mix strategy implies that, for an investment proportion $m>1(m<1)$, additional asset are bought (sold) if the asset price increases. In particular, for $m>1$ the resulting payoff is convex in the asset price so that a constant mix strategy can, at least technically, be classified as a portfolio insurance strategy. However, the payoff is floored by zero. In theory, it is straightforward to achieve optimal strategies yielding payoffs with a positive floor. Here, the expected utility is maximized under the additional constraint that the terminal portfolio value must be above a strictly positive terminal guarantee. Alternatively, the floor can be achieved if the utility is measured in terms of the difference of the portfolio value and the guarantee instead of the portfolio wealth itself, i.e. if a utility function with a subsistence level is used.

The modified optimization problems help to understand the most prominent approaches of portfolio insurance strategies, i.e. CPPI and OBPI strategies. The modifications which are imposed on the unconstraint optimization problem give interesting modifications for the payoffs. Using the unrestricted optimization problem as benchmark, the constant mix strategy and its associated payoff with floor zero defines also a benchmark for OBPI and CPPI strategies. The CPPI results from a subsistence level, i.e. the utility is measured in terms of the difference of portfolio value and guarantee instead of the portfolio value itself. In contrast, the OBPI results from the additional constraint that the terminal payoff is above the guarantee or floor. Considering the associated payoffs, both approaches result in payoffs which consist of a fraction of the payoff of a constant mix strategy and an additional term stemming from the guarantee. Intuitively, it is clear that the fraction is linked to the price of the guarantee, i.e. the fraction is less than one. The main difference between OBPI and CPPI can easily be explained by the additional term. In the case of the CPPI approach, the additional term is simply the guarantee itself, i.e. the payoff of the adequate number of zero bonds. In contrast, the additional term implied by the OBPI is based on a put on the fraction of constant mix payoffs with strike equal to the guarantee. Obviously, the put on the zero coupon bond is cheaper than the zero bond 
itself. Thus, the OBPI fraction which is held of the optimal unrestricted payoff is higher than in the case of the CPPI method. This is a major advantage in terms of the associated utility costs, i.e. the loss in expected utility which is caused by the introduction of a strictly positive guarantee. The utility costs are measured and illustrated in terms of a loss rate linking the certainty equivalents of the strict portfolio insurance strategies to the certainty equivalent of the optimal solution.

One major drawback of the OBPI method is due to its kinked payoff-profile. The terminal value of the OBPI is equal to the guarantee if the put expires out of the money. In contrast to the CPPI method, this implies a positive point mass that the terminal value is equal to the guarantee. This relevant probability is given by the real world probability that the terminal asset prices is below the strike of the put. Intuitively, it is clear that this can cause a high exposure to gap risk, i.e. the risk that the guarantee is not honored, if market frictions are introduced. We illustrate this effect by taking trading restrictions and transaction costs into account. It turns out that the guarantee implied by the CPPI method is relatively robust. However, the probability that the guarantee is not reached under the corresponding synthetic discrete-time OBPI strategy is rather high.

Finally, we tackle the question of borrowing constraints. In a strict sense, borrowing constraints imply that the proportion of asset must not be above one. In the case of the CPPI method, the capped CPPI which simply states that the asset proportion is adequately capped according to the borrowing constraints is of high practical relevance. We give the distribution of the capped CPPI and illustrate the corresponding loss rate, i.e. the loss which is due to borrowing constraints. 


\section{REFERENCES}

Ahn, D.-H., Boudoukh, Richardson, M. and Whitelaw, R. (1999), Optimal Risk Management Using Options, Journal of Finance LIV(1), 359-375.

Avellaneda, M., Levy, A. and Parás, A. (1995), Pricing and Hedging Derivative Securities in Markets with Uncertain Volatilities, Applied Mathematical Finance 2(2), 73-88.

Balder, S. and Mahayni, A. (2008), Cash-Lock Comparison of Portfolio Insurance Strategies, Technical reprot.

Balder, S., Brandl, M. and Mahayni, A. (2009), Effectiveness of CPPI Strategies und Discrete-Time Trading, The Journal of Economic Dynamics and Control 33, 204-220.

Basak, S. (1995), A General Equilibrium Model of Portfolio Insurance, Review of Financial Studies 8(4), 1059-1090.

Basak, S. (2002), A Comparative Study of Portfolio Insurance, The Journal of Economic Dynamics and Control 26(7-8), 1217-1241.

Benninga, S. and Blume, M. (1985), On the Optimality of Portfolio Insurance, Journal of Finance 40(5), 1341-1352.

Bergman, Y., Grundy, B. and Wiener, Z. (1996), General Properties of Option Prices, Journal of Finance 51, 1573-1610.

Bertrand, P. and Prigent, J.-L. (2002a), Portfolio Insurance Strategies: OBPI versus CPPI, Technical report, GREQAM and Université Montpellier1.

Bertrand, P. and Prigent, J.-L. (2002b), Portfolio Insurance: The Extreme Value Approach to the CPPI, Finance.

Bertrand, P. and Prigent, J.-L. (2003), Portfolio Insurance Strategies: A Comparison of Standard Methods When the Volatility of the Stoch is Stochastic, International Journal of Business 8(4), 15-31.

Black, F. and Jones, R. (1987), Simplifying Portfolio Insurance, The Journal of Portfolio Management 14, 48-51.

Black, F. and Perold, A. (1992), Theory of Constant Proportion Portfolio Insurance, The Journal of Economic Dynamics and Control 16(3-4), 403-426.

Bookstaber, R. and Langsam, J. (2000), Portfolio Insurance Trading Rules, The Journal of Futures Markets 8, 15-31.

Brennan, M. and Schwartz, E. (1976), The Pricing of Equity-Linked Life Insurance Policies with an Asset Value Guarantee, Journal of Financial Economics 3, 195-213.

Brennan, M. and Schwartz, E. (1989), Portfolio Insurance and Financial Market Equilibrium, Journal of Business 62, 455-472.

Browne, S. (1999), Beating a Moving Target: Optimal Portfolio Strategies for Outperforming a Stochastic Benchmark, Finance and Stochastics 3, 275-294.

Cesari, R. and Cremonini, D. (2003), Benchmarking, portfolio insurance and technical 
analysis: a Monte Carlo comparison of dynamic strategies of asset allocation, Journal of Economic Dynamics and Control 27, 987-1011.

Cont, R. and Tankov, P. (2007), Constant Proportion Portfolio Insurance in Presence of Jumps in Asset Prices, Financial Engigeering No 2007-10, Columbia University Center for Financial Engigeering.

Cox, C. and Leland, H. (2000), On dynamic investment strategies, The Journal of Economic Dynamics and Control 24, 1859-1880.

Cox, J. and Huang, C.-F. (1989), Optimal Consumption and Portfolio Policies when the Asset Price follows a Diffusion Process, Journal of Economic Theory 49, 33-83.

Doskeland, T. M. and Nordahl, H. A. (2008), Optimal pension insurance design, Journal of Banking and Finance 32, 382-392.

Dudenhausen, A., Schlögl, E. and Schlögl, L. (1998), Robustness of Gaussian Hedges under Parameter and Model Misspecification, Technical report, University of Bonn, Department of Statistics.

El Karoui, N., Jeanblanc, M. and Lacoste, V. (2005), Optimal Portfolio Management with American Capital Guarantee, Journal of Economic Dynamics and Control 29, 449-468.

El Karoui, N., Jeanblanc-Picqué, M. and Shreve, S. (1998), Robustness of the Black and Scholes Formula, Mathematical Finance 8(2), 93-126.

Esser, A. (2003), General Valuation Principles for Arbitrary Payoffs and Applications to Power Options under Stochastic Volatility Models, Financial Markets and Portfolio Management 17, 351-372.

Grossman, S. and Villa, J. (1989), Portfolio Insurance in Complete Markets: A Note, Journal of Business 62, 473-476.

Grossman, S. and Zhou, J. (1993), Optimal Investment Strategies for Controlling Drawdowns, Mathematical Finance 3, 241-276.

Grossman, S. and Zhou, J. (1996), Equilibrium Analysis of Portfolio Insurance, Journal of Finance 51, 1379-1403.

Hobson, D. (1998), Volatility Misspecification, Option Pricing and Superreplication via Coupling, Annals of Applied Probability 8(1), 193-205.

Kahnemann, D. and Tversky, A. (1979), Prospect Theory: An Analysis of Decision under Risk, Econometrica 47, 263-291.

Leland, H. (1980), Who should buy Portfolio Insurance, Journal of Finance 35(2), 581594.

Leland, H. (1988), Portfolio Insurance and October $19^{\text {th }}$, California Management Review Summer, 80-89.

Leland, H. and Rubinstein, M. (1976), The Evolution of Potfolio Insurance, in: D.L. 
Luskin, ed., Portfolio Insurance: A guide to Dynamic Hedging, Wiley.

Lyons, T. (1995), Uncertain Volatility and the Risk-free Synthesis of Derivatives, Applied Mathematical Finance 2, 117-133.

Mahayni, A. (2003), Effectiveness of Hedging Strategies under Model Misspecification and Trading Restrictions, International Journal of Theoretical and Applied Finance 6(5), 521-552.

Mahayni, A. and Schlögl, E. (2008), The Risk Management of Minimum Return Guarantees, Business Research 1, 55-76.

Merton, R. (1971), Optimal Consumption and Portfolio Rules in a Continuous Time Model, Journal of Economic Theory 3, 373-413.

Munk, C. (2008), Financial asset pricing theory, Lecture notes.

Tepla, L. (2000), Optimal portfolio policies with borrowing and shortsale constraints, The Journal of Economic Dynamics and Control 24, 1623-1639.

Tepla, L. (2001), Optimal Investment with Minimum Performance Constraints, The Journal of Economic Dynamics and Control 25(10), 1629-1645.

Tversky, A. and Kahnemann, D. (1992), Advances in Prospect Theory: Cumulative Representation of Uncertainty, Journal of Risk and Uncertainty 5, 297-323.

von Neumann, J. and Morgenstern, O. (1944), Theory of Games and Economic Behavior, 1953 ed.. Princeton University Press, Princeton, NJ.

Zagst, R. and Kraus, J. (2008), Stochastic Dominance of Portfolio Insurance Strategies - OBPI versus CPPI, Technical reprot.

Zhang, P. G. (1998), Exotic Options-A Guide to Second Generation Options, World Scientific. 